QMUL-PH-08-12

\title{
Diagonal free field matrix correlators, global symmetries and giant gravitons
}

\author{
T.W. Brown ${ }^{1}$, P.J. Heslop ${ }^{2}$ and S. Ramgoolam ${ }^{3}$ \\ Centre for Research in String Theory, Department of Physics \\ Queen Mary, University of London \\ Mile End Road, London E1 4NS, UK
}

\begin{abstract}
We obtain a basis of diagonal free field multi-matrix 2-point correlators in a theory with global symmetry group $G$. The operators fall into irreducible representations of $G$. This applies for gauge group $U(N)$ at finite $N$. For composites made of $n$ fundamental fields, this is expressed in terms of Clebsch-Gordan coefficients for the decomposition of the $n$-fold tensor products of the fundamental field representation in terms of $G \times S_{n}$ representations. We use this general construction in the case of the $S L(2)$ sector of $\mathcal{N}=4$ SYM. In this case, by using oscillator constructions, we reduce the computation of the relevant Clebsch-Gordans coupling infinite dimensional discrete series irreps of $S L(2)$ to a problem in symmetric groups. Applying these constructions we write down gauge invariant operators with a Fock space structure similar to that arising in a large angular momentum limit of worldvolume excitations of giant gravitons. The Fock space structure emerges from Clebsch multiplicities of tensor products of symmetric group representations. We also give the action of the 1-loop dilatation operator of $\mathcal{N}=4 \mathrm{SYM}$ on this basis of multi-matrix operators.
\end{abstract}

\footnotetext{
${ }^{1}$ E-mail address: t.w.brown@qmul.ac.uk

${ }^{2}$ E-mail address: p.j.heslop@qmul.ac.uk

${ }^{3}$ E-mail address: s.ramgoolam@qmul.ac.uk
} 


\section{Contents}

1 Introduction

1.1 Schur-Weyl duality and $G \times S_{n}$ Clebsch decompositions

1.2 Outline of paper and main results

$2 S L(2)$ sector: covariant operators

2.1 Oscillator construction

2.2 Action of $S_{n}$ on $A_{a}$

2.3 Canonical covariant form using oscillators

2.4 Descendants

3 Multiplicities of $S L(2) \times S_{n}$ irreps. in $V_{F}^{\otimes n}$

3.1 Multiplicity of $S L(2)$ irreps.

3.2 Multiplicities of $S L(2) \times S_{n}$ and $q$-deformed $G L(\infty)$

3.2.1 Examples of symmetric and antisymmetric $S_{n}$ irreps .

3.2.2 The generating function for any $S L(2) \times S_{n}$ irreps

3.2.3 Proof using $q$-dimensions of $G L(\infty)$

3.3 The general case using characters of symmetric groups $S_{n} \times S_{k}$

4 General $G, V$

4.1 Correlators in free field theory . . . . . . . . . . . . . . . . . 16

4.1.1 Signs in the super-algebra case . . . . . . . . . . . . . . . . . . . . . . . . . . . .

4.2 Completeness ................................ 18

5 Examples and applications

$5.1 S L(2)$ and $\operatorname{Sym}\left(V_{H}^{\otimes k}\right) \ldots \ldots \ldots \ldots \ldots \ldots \ldots \ldots$

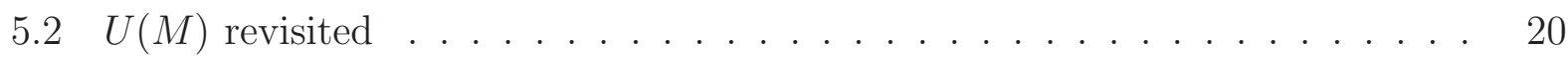

5.3 The higher $\operatorname{spin}$ group . . . . . . . . . . . . . . . . . . . . . . . . . . . . . . . . . .

5.4 The $S O(6)$ sector $\ldots \ldots \ldots \ldots \ldots \ldots \ldots \ldots \ldots$

5.5 The $S O(4,2)$ sector . . . . . . . . . . . . . . . . . . . . . . . . . . . . . . . . . . . .

5.6 Fields carrying reps of product groups . . . . . . . . . . . . . . . . . . . . . . . . . . . . . . . . .

5.6.1 Product Clebsch in terms of single group Clebschs . . . . . . . . . 23

6 Gauge invariant operators 24

6.1 Finite $N$ counting . . . . . . . . . . . . . . . . . . 25

7 Worldvolume excitations of giants and gauge invariant operators 27

7.1 Worldvolume excitations: review and comments . . . . . . . . . . . . . . 27

7.2 Comparison to gauge invariant operators . . . . . . . . . . . . . . . . . . . . . . . . . . . . . . . . . . . . .

7.3 Comments . . . . . . . . . . . . . . . . . . . . . . . . . .

8 One-loop mixing in $\mathcal{N}=4$ 
A Clebsch-Gordan decomposition for $V_{H}^{\otimes k}$

A.1 Multiplicities from characters . . . . . . . . . . . . . . . 36

A.2 Clebsch-Gordan coefficients . . . . . . . . . . . . . . . . 36

A.3 Symmetrised Clebsch from ordinary Clebsch . . . . . . . . . . . . . . . . . . . . . . . . . . .

A.4 Symmetrised Clebsch multiplicities . . . . . . . . . . . . . . . 38

B Action of $S_{n}$ on $A_{a}^{\dagger}$ in detail 39

\begin{tabular}{ll} 
C Code & 39 \\
\hline
\end{tabular}

\section{Introduction}

AdS/CFT [1, 2, 3] allows a translation of many hard questions of quantum gravity to questions in the dual CFT. Intriguing lessons on quantum gravity have taken the form of the stringy exclusion principle [4]. Some aspects of this principle find a geometrical expression in the properties of giant gravitons [5, 6]. The systematic study of the correlation functions of gauge invariant multi-trace local operators in the half-BPS sector has been fruitful in identifying CFT duals [7, 8, 9] of giant gravitons, strings attached to them [10, 11, 12] as well as bulk geometries resulting from their back-reaction on space-time [13. An important step in these maps to spacetime is the identification of appropriate diagonal bases in the space of multi-trace operators. For holomorphic operators constructed from a single complex matrix, relevant to the half-BPS sector, this was solved in [8. Recent generalizations including non-holomorphic operators and multi-matrices have been achieved [14, 15, 16].

In [15] we solved the diagonalisation in the sector of holomorphic operators constructed from $M$ complex matrices transforming in the fundamental of $U(M)$ (the case of $M=3$ being of interest in $\mathcal{N}=4 \mathrm{SYM}$ ). We gave covariant operators with correlators of the simple form

$$
\left\langle\left(\hat{\mathcal{O}}^{\Lambda, M_{\Lambda}, i}\right)_{J}^{I}\left(\hat{\mathcal{O}}^{\dagger \Lambda^{\prime}, M_{\Lambda^{\prime}}^{\prime}, i^{\prime}}\right)_{L}^{K}\right\rangle=\delta^{\Lambda \Lambda^{\prime}} \delta^{M_{\Lambda} M_{\Lambda^{\prime}}^{\prime}} \sum_{\sigma \in S_{n}} D_{i i^{\prime}}^{\Lambda}(\sigma) \quad(\sigma)_{J}^{K}\left(\sigma^{-1}\right)_{L}^{I}
$$

We will call this the canonical covariant form. $\Lambda$ is the $U(M)$ irreducible representation and $M_{\Lambda}$ labels the state within this irrep. $D_{i i^{\prime}}^{\Lambda}(\sigma)$ is the orthogonal matrix representation of the $S_{n}$ representation $\Lambda$. There is no spacetime dependence because we are considering a $4 \mathrm{~d}$ analogue of the Zamolodchikov metric used in 2d CFT. We will often use the expression 'two-point function' interchangeably with this metric.

These covariant operators lead to gauge invariant operators with diagonal correlation functions

$$
\left\langle\mathcal{O}^{\Lambda, M_{\Lambda}, R, \tau} \mathcal{O}^{\dagger \Lambda^{\prime}, M_{\Lambda^{\prime}}^{\prime}, R^{\prime}, \tau^{\prime}}\right\rangle \propto \delta^{\Lambda \Lambda^{\prime}} \delta^{M_{\Lambda} M_{\Lambda^{\prime}}^{\prime}} \delta^{R R^{\prime}} \delta^{\tau \tau^{\prime}}
$$

$R$ is the $U(N)$ representation which organises the multi-trace structure of the operator. 
In this paper we will explain how to get the canonical covariant form (11) for any global symmetry group $G$. This will use Schur-Weyl duality which we review below. The diagonal operators for the gauge invariant operators (2) follows automatically (see Section 6).

Applied to $\mathcal{N}=4 \mathrm{SYM}$ this means we solve the problem of writing down a basis for the space of all gauge invariant operators in the theory (and in any subsector of the theory). Operators are labelled by lowest weight representations of the global symmetry group and diagonalise the free two-point correlation functions. We write these operators in terms of appropriate Clebsch-Gordon coefficients and it should be noted that finding the CG coefficients themselves is still a difficult problem in general, but we show how to do this in the particular example of the $S L(2)$ sector.

\subsection{Schur-Weyl duality and $G \times S_{n}$ Clebsch decompositions}

Let $V_{F}$ be the fundamental representation of $U(M)$ (or $G L(M)$ ). Classical Schur-Weyl duality gives the decomposition of $V_{F}^{\otimes n}$ in terms of irreducible representations of $U(M)$. It relies on the fact that in the algebra of linear operators acting $V_{F}^{\otimes n}$, i.e. in $\operatorname{End}\left(V_{F}^{\otimes n}\right)$, the maximal subalgebra which commutes with $U(M)$ is exactly the group algebra of $S_{n}$. It gives the decomposition of $V_{F}^{\otimes n}$ under $U(M) \otimes S_{n}$ :

$$
V_{F}^{\otimes n}=\bigoplus_{\Lambda \vdash n} V_{\Lambda}^{U(M)} \otimes V_{\Lambda}^{S_{n}}
$$

Here $\Lambda \vdash n$ denotes the fact that $\Lambda$ runs over partitions of $n$, which correspond to Young diagrams with row lengths $\left(r_{1}, r_{2} \cdots\right)$ with $r_{i} \geq r_{i+1}$. According to (3), $V_{F}^{\otimes n}$ has a complete basis of states of the form $\left|\Lambda, M_{\Lambda}, m_{\Lambda}\right\rangle$, where $M_{\Lambda}$ label states in the irrep. of $U(M)$ corresponding to the Young diagram $\Lambda$ and $m_{\Lambda}$ label states in the irrep. of $S_{n}$ corresponding to the same Young diagram. For $n>M$, the above has to be qualified with the constraint that $c_{1}(\Lambda) \leq M$, i.e. the maximal length of the first column of the Young diagram is $M$.

Schur-Weyl duality is a special case of the double centraliser theorem (or double commutant theorem) which gives a decomposition generalising (3) for any algebra acting on a vector space $\mathcal{W}$ (for a brief statement of the key relevant facts see Section 1 of [17] or textbooks such as [18]). The decomposition is given in terms of the algebra of interest $A$ and the commutant (centraliser) of its action in the vector space, often denoted as $\operatorname{End}_{A}(\mathcal{W})$. Let $G$ be the global symmetry group of a field theory. Let $V_{F}$ be a representation of $G$ formed by a set of fields in the theory. We will consider $\mathcal{W}=V_{F}^{\otimes n}$ and the algebra of interest is the universal enveloping algebra of the Lie algebra of $G$. Often $G$ will be a subgroup of the full symmetry group of a gauge theory. In the case of $\mathcal{N}=4 \mathrm{SYM}$, if we are interested in the six hermitian scalars (without space-time derivatives), $V_{F}$ is the fundamental of $S O(6)$. If we write the six hermitian scalars as three complex ones, and only consider the holomorphic combinations, then the symmetry group of interest is $U(3)$ and $V_{F}$ is the fundamental 3-dimensional representation. This is the case we studied in [15] and is covered by classical Schur-Weyl duality. In this paper one of the main examples will involve $G=S L(2)$ and $V_{F}$ will be an infinite 
dimensional discrete series representation corresponding to a single scalar field $X$ and its derivatives with respect to one light-cone direction, $\partial X, \partial^{2} X, \cdots$. More generally we have fields $W_{m} \in V_{F}$ where $m$ may run over an infinite dimensional vector space. In the $S L(2)$ example we would have $W_{m}=\partial^{m} \Phi$. We will then consider the $n$-field composites

$$
W_{m_{1}} \otimes W_{m_{2}} \otimes \cdots \otimes W_{m_{n}}
$$

which transform in the representation $V_{F}^{\otimes n}$ of $G$. This space also has an action of the symmetry group $S_{n}$ which permutes the $n$ fields. This $S_{n}$ action commutes with $G$, but does not provide the full commutant of $G$. Equivalently there is a non-trivial commutant algebra of $G \times S_{n}$, denoted by $\operatorname{Com}\left(G \times S_{n}\right)$. By the double-commutant theorem, this will organise the multiplicities of $G \times S_{n}$ representations. We have

$$
\left(V_{F}^{G}\right)^{\otimes n}=\bigoplus_{\Lambda, \Lambda_{1}} V_{\Lambda}^{G} \otimes V_{\Lambda_{1}}^{S_{n}} \otimes V_{\Lambda, \Lambda_{1}}^{\operatorname{Com}\left(G \times S_{n}\right)}
$$

where $\Lambda$ is a representation of the global group $G$ and $\Lambda_{1}$ is a representation of $S_{n}$. $V_{\Lambda, \Lambda_{1}}$ gives the multiplicity with which $\Lambda$ of $G$ and $\Lambda_{1}$ of $S_{n}$ appear together. It is a representation of $\operatorname{Com}\left(G \times S_{n}\right)$. The explicit form of $\operatorname{Com}\left(G \times S_{n}\right)$ will not be needed in this paper, with $V_{\Lambda, \Lambda_{1}}$ appearing simply as the carrier space of a multiplicity label. In diagonalising the free-field two point functions, the decomposition of $V_{F}^{\otimes n}$ in terms of the group $G \times S_{n}$ will be crucial.

The study of the multiplicity of the decomposition of $n$-fold tensor powers in terms of $G \times S_{n}$ is called the plethysm problem. This is solved for $S U(2)$ in [19] and is, for more general $G$, the subject of a large mathematical literature. For developments on the use of the combinatorics related to plethysms in the context of chiral rings of a large class of $\mathcal{N}=1$ SYM theories see [20]. For our purposes, we will be interested, not only in the multiplicities of plethysms but also the corresponding Clebsch-Gordan coefficients. Using these we will obtain explicit gauge invariant operators which diagonalize the Zamolodchikov metric.

\subsection{Outline of paper and main results}

In Section 2 we will find the canonical covariant form for the $S L(2)$ sector. In particular we will have in mind derivatives acting on one complex scalar $X$. In Section 3, we will give explicit formulae for the $S L(2) \times S_{n}$ multiplicities involved. We will be heavily using the oscillator construction of $S L(2)$. In Section 4, we show, for any global symmetry $G$ and for $n$-field composites with the basic fields transforming in any representation $V$ of $G$, that the covariant canonical form follows once we construct $n$-field composites using the Clebsch-Gordan coefficients for the $G \times S_{n}$ decomposition of $V^{\otimes n}$. In Section 5 we give various examples of this general case explaining how the diagonalisations in the $U(M)$ and $S L(2)$ sectors follow this general pattern, and how the canonical covariant corresponding to representations of the higher spin group $H S(1,1)$ are also included. We describe some useful facts concerning $S O(6) \times S_{n}$ multiplicities relevant to the $S O(6)$ sector of six hermitian scalars and finally we show how to apply the construction of Section 4 to the case where 
where $G$ is of the product form $G_{1} \times G_{2}$. In Section 6 we review how to get the diagonal gauge-invariant operators from the canonical covariant form and show the compatibility of the counting with matrix model methods of [21]. Section 7 shows how our results on the $S L(2)$ sector can be used to provide the gauge theory operators dual to the worldvolume excitations of giants considered in [22]. The one-loop mixing of the gauge-invariant operators constructed in Section 6 for $\mathcal{N}=4$ is analysed in Section 8 .

\section{$2 \quad S L(2)$ sector: covariant operators}

We consider the $S L(2)$ sector which we can view as a reduction of $\mathcal{N}=4 \mathrm{SYM}$ to a sector with a single light-cone derivative of the complex scalar $X$. We choose $\partial \equiv\left(\partial_{0}+\partial_{3}\right) / 2$.

We find the basic two-point function

$$
\left\langle\partial^{k_{1}} X_{j}^{\dagger i}(x) \partial^{k_{2}} X_{l}^{k}(0)\right\rangle=\frac{(-1)^{k_{1}}\left(k_{1}+k_{2}+1\right) !}{x^{2+k_{1}+k_{2}}} \delta_{l}^{i} \delta_{j}^{k}
$$

If we consider $\mathcal{N}=4 \mathrm{SYM}$ on $\mathcal{R}^{4}$, we take our two operators to zero and infinity (corresponding to opposite poles of the conformally equivalent $S^{4}$ ) we have

$$
\left\langle\partial^{k_{1}} X_{j}^{\dagger / i}\left(x^{\prime}=0\right) \partial^{k_{2}} X_{l}^{k}(x=0)\right\rangle=\delta^{k_{1} k_{2}}\left(k_{1} !\right)^{2} \delta_{l}^{i} \delta_{j}^{k}
$$

where $x^{\prime}=x / x^{2}$ is the coordinate patch around the north pole and $x$ around the south. This technique is well known from the studies of conformal field theories in two dimensions and the above is known as the Zamolodchikov metric (see [23, 24] for a general account and 25] for applications to $\mathcal{N}=4 \mathrm{SYM}$ ). Note that this metric on operators is defined using space-time dependent two-point functions but is itself independent of spacetime. Knowing the metric for arbitrary derivatives allows a reconstruction of the spacetime dependence.

\section{$2.1 \quad$ Oscillator construction}

The oscillator representation allows an elegant method of constructing primary fields in the $S L(2)$ sector [26, 27]. By using this representation to find the Clebsch-Gordan coefficients associated with the $S L(2) \times S_{n}$, we will solve the problem of finding the canonical covariant 2-point functions. It will turn out that in addition to the groups $S L(2)$ and $S_{n}$ another symmetric group will play an interesting role. It is $S_{k}$ where $k$ is the number of derivatives required to construct the lowest weight state.

The $S O(4,2)$ conformal algebra is given by

$$
\begin{aligned}
{\left[M_{a b}, P_{c}\right] } & =\eta_{b c} P_{a}-\eta_{a c} P_{b}, \quad\left[M_{a b}, K_{c}\right]=\eta_{b c} K_{a}-\eta_{a c} K_{b}, \\
{\left[M_{a b}, M_{c d}\right] } & =\eta_{b c} M_{a d}-\eta_{a c} M_{b d}+\eta_{a d} M_{b c}-\eta_{b d} M_{a c}, \\
{\left[D, P_{a}\right] } & =P_{a}, \quad\left[D, K_{a}\right]=-K_{a}, \quad\left[K_{a}, P_{b}\right]=2 \eta_{a b} D-2 M_{a b}
\end{aligned}
$$


The $S L(2)$ sector in terms of the conformal generators can be chosen as

$$
L_{+}=\frac{1}{2}\left(P_{0}+P_{3}\right) \quad L_{-}=\frac{1}{2}\left(K_{0}-K_{3}\right) \quad L_{0}=\frac{1}{2}\left(D-M_{03}\right)
$$

giving

$$
\left[L_{-}, L_{+}\right]=2 L_{0}, \quad\left[L_{0}, L_{ \pm}\right]= \pm L_{ \pm}
$$

This algebra may be represented using oscillators as

$$
L_{+}=a^{\dagger}+a^{\dagger} a^{\dagger} a, \quad L_{0}=\frac{1}{2}+a^{\dagger} a, \quad L_{-}=a
$$

where $\left[a, a^{\dagger}\right]=1$. The lowest weight state of the representation $V_{F}$ is denoted $|0\rangle$ and is annihilated by all the lowering oscillators $L_{-}=a$. It can straightforwardly be checked that the raising operators $L_{+}$then act on the lowest weight state as

$$
\left(L_{+}\right)^{k}|0\rangle=k !\left(a^{\dagger}\right)^{k}|0\rangle \quad \leftrightarrow \quad \partial^{k} X|0\rangle
$$

By the operator-state correspondence, the operator on the RHS above acts on the CFT vacuum at the origin in radial quantization to give a state. Hence we have a map from oscillator states used in the representation theory of $S L(2)$ to states in radial quantization. Dual states in the oscillator Hilbert space map to states at the dual vacuum (at infinity) in radial quantization.

$$
\langle 0| L_{-}^{k}=\langle 0| a^{k} \quad \leftrightarrow \quad\langle 0| \partial^{k} X
$$

Note that the usual oscillator inner product correctly maps to the inner product given by the Zamolodchikov metric (7). The normalization in (77) is easily calculated using the $S L(2)$ algebra once we use the fact that $L_{-}$is the hermitian conjugate of $L_{+}$in radial quantization.

In a similar way we can represent the tensor product $V_{F}^{\otimes n}$ by considering $n$ independent oscillators $a_{i}$. In this space the action of the diagonal $S L(2)$ is obtained by summing over $n$ :

$$
\mathbf{L}_{+}=\sum_{i}\left(a_{i}^{\dagger}+a_{i}^{\dagger} a_{i}^{\dagger} a_{i}\right), \quad \mathbf{L}_{0}=\frac{1}{2} n+\sum_{i} a_{i}^{\dagger} a_{i}, \quad \mathbf{L}_{-}=\sum_{i} a_{i}
$$

The relation between the oscillator states and the field states is:

$$
\prod_{l=1}^{n}\left(a_{l}^{\dagger}\right)^{k_{l}}|0\rangle \quad \leftrightarrow \quad \frac{1}{k_{1} ! k_{2} ! \ldots k_{n} !} \partial^{k_{1}} X \otimes \partial^{k_{2}} X \otimes \cdots \otimes \partial^{k_{n}} X \quad|0\rangle
$$

The lowest weights are annihilated by $L_{-}=\sum_{i} a_{i}$. All the lowest weight states at level $L_{0}=n+k$ are generated by $k$-oscillator states obtained as products of $\left(a_{i}^{\dagger}-a_{j}^{\dagger}\right)$ acting on the vacuum. The simplest example is at $n=2$ where the lowest weight states are all of the form

$$
\mathcal{O}_{k}=\left(a_{1}^{\dagger}-a_{2}^{\dagger}\right)^{k}|0\rangle
$$


Expanding out the oscillators and using (17) we find the corresponding operators in field space

$$
\mathcal{O}_{k} \sim \sum_{j=0}^{k}\left(\begin{array}{l}
k \\
j
\end{array}\right)^{2}(-1)^{k-j} \partial^{j} X \otimes \partial^{k-j} X
$$

These are conformal higher spin currents, first constructed in [28].

\subsection{Action of $S_{n}$ on $A_{a}$}

The lowest weight states are clearly generated by the $n-1$ differences of oscillators $a_{i}^{\dagger}-a_{j}^{\dagger}$. The following basis will be very useful

$$
A_{a}^{\dagger}=\sum_{i=1}^{n} J_{a}{ }^{i} a_{i}^{\dagger}
$$

where $J_{a}{ }^{i}$ takes us from the natural representation of $S_{n}$ on $n$ objects (labelled by the index $i$ ) to the $n-1$ dimensional $H=[n-1,1]$ representation for which we will choose the orthonormal basis (labelled by the index $a$ ) 1 . The matrix $J$ will thus have the following properties

$$
\begin{aligned}
D_{a b}^{H}(\sigma) J_{b}{ }^{i} & =J_{a}{ }^{\sigma(i)} \\
J_{a}{ }^{i}\left(J^{\dagger}\right)_{b}{ }^{i} & =\delta_{a b}
\end{aligned}
$$

with $D_{a b}^{H}(\sigma)$ the orthonormal representation. Explicitly we find

$$
A_{a}^{\dagger}=\frac{1}{\sqrt{i(i+1)}}\left(a_{1}^{\dagger}+\ldots a_{a}^{\dagger}-i a_{a+1}^{\dagger}\right)
$$

The details of the $S_{n}$ action on $A_{a}^{\dagger}$, and its relation to the orthogonal representing matrix of the hook representation $D_{a b}^{H}(\sigma)$, are given in Appendix Section B.

\subsection{Canonical covariant form using oscillators}

The simplest operator in the space $V_{F}^{\otimes n}$ is given by $n$ scalar fields without derivatives

$$
\left(\mathcal{O}_{n}\right)_{j_{1} j_{2} \cdots j_{n}}^{i_{1} i_{2} \cdots i_{n}}=X_{j_{1}}^{i_{1}} \otimes X_{j_{2}}^{i_{2}} \otimes \cdots \otimes X_{j_{n}}^{i_{n}}
$$

The scalar fields lie in the adjoint representation of the gauge group $U(N)$. Using multiindices $I=\left(i_{1}, i_{2} \cdots i_{n}\right)$ and $J=\left(j_{1}, j_{2} \cdots j_{n}\right)$ we can write

$$
\left(\mathcal{O}_{n}\right)_{J}^{I}=(X \otimes X \otimes \cdots X)_{J}^{I} \equiv \mathbf{X}_{J}^{I}
$$

\footnotetext{
${ }^{1}$ The natural representation of $S_{n}$ on $n$ objects is a reducible representation and decomposes into the $n-1$ dimensional rep $H=[n-1,1]$ and the 1-dimensional symmetric rep $[n]$. We consider the map from $a_{i}$ onto the symmetric representation in Section 2.4 where we see that they correspond to the application of the $S L(2)$ descendant operator.
} 
There is a map which takes the states in the oscillator construction of the $V_{F}^{\otimes n}$ representation of $S L(2)$ to operators or states (by the operator-state correspondence) in the CFT. The vacuum of the oscillator construction maps to $\mathbf{X}_{J}^{I}|0\rangle$. Denoting the map from oscillator Hilbert space to the CFT states as $\rho_{J}^{I}$ we may write

$$
\rho_{J}^{I}(|0\rangle) \leftrightarrow \mathbf{X}_{J}^{I}|0\rangle \equiv\left|\mathbf{X}_{J}^{I}\right\rangle
$$

The vacuum on the right can be viewed as the vacuum in the CFT associated with the origin in radial quantization. The dual oscillator vacuum maps to the dual vacuum at infinity in radial quantization.

$$
\rho_{J}^{I}(\langle 0|) \leftrightarrow\langle 0| \mathbf{X}_{J}^{\dagger} \equiv\left\langle\mathbf{X}_{J}^{I}\right|
$$

The correlation function of these operators is

$$
\left\langle\mathbf{X}_{J}^{I} \mid \mathbf{X}_{L}^{K}\right\rangle=\sum_{\sigma \in S_{n}} \sigma_{L}^{I}\left(\sigma^{-1}\right)_{J}^{K}
$$

Now the operator corresponding to $a_{i}^{\dagger}|0\rangle$ has $n-1$ scalar fields $X$ with one derivative of a scalar field $\partial X$ sitting at position $i$. Summing over Wick contractions and using the orthogonality in (7) gives the following correlation function

$$
\rho_{J}^{I}\left(\langle 0| a_{i}\right) \rho_{L}^{K}\left(a_{j}^{\dagger}|0\rangle\right)=\sum_{\sigma \in S_{n}} \delta_{i \sigma(j)} \sigma_{L}^{I}\left(\sigma^{-1}\right)_{J}^{K}
$$

Here one only sums over permutations $\sigma$ which map position $j$ to $i$, enforced by the delta function. This in turn leads to the correlation function for $k$ oscillators ( $k$ derivatives):

$$
\rho_{J}^{I}\left(\left\langle 0\left|a_{i_{1}} a_{i_{2}} \ldots a_{i_{k}}\right|\right) \rho_{L}^{K}\left(a_{j_{1}}^{\dagger} a_{j_{2}}^{\dagger} \ldots a_{j_{k}}^{\dagger}|0\rangle\right)=\sum_{\rho \in S_{k}} \sum_{\sigma \in S_{n}} \delta_{i_{1} \sigma\left(j_{\rho(1)}\right)} \ldots \delta_{i_{k} \sigma\left(j_{\rho(k)}\right)} \sigma_{L}^{I}\left(\sigma^{-1}\right)_{J}^{K}\right.
$$

We wish to rewrite this in terms of the lowest weight states $\rho_{L}^{K}\left(A_{a_{1}}^{\dagger} A_{a_{2}}^{\dagger} \ldots A_{a_{k}}^{\dagger}|0\rangle\right)$. The correlation function of these states is then

$$
\begin{aligned}
& \rho_{J}^{I}\left(\langle 0|\left(A_{a_{1}} A_{a_{2}} \ldots A_{a_{k}}\right) \rho_{L}^{K}\left(A_{b_{1}}^{\dagger} A_{b_{2}}^{\dagger} \ldots A_{b_{k}}^{\dagger}|0\rangle\right)\right. \\
& =\sum_{\rho \in S_{k}} \sum_{\sigma \in S_{n}} J_{a_{1}}^{\sigma\left(j_{\rho(1)}\right)} \ldots J_{a_{k}}^{\sigma\left(j_{\rho(k)}\right)}\left(J^{\dagger}\right)_{b_{1}}^{j_{1}} \ldots\left(J^{\dagger}\right)_{b_{k}}^{j_{k}} \sigma_{L}^{I}\left(\sigma^{-1}\right)_{J}^{K} \\
& =\sum_{\rho \in S_{k}} \sum_{\sigma \in S_{n}} D_{a_{1} c_{1}}^{H}(\sigma) \ldots D_{a_{k} c_{k}}^{H}(\sigma) J_{c_{1}}^{j_{\rho(1)}} \ldots J_{c_{k}}^{j_{\rho(k)}}\left(J^{\dagger}\right)_{b_{1}}^{j_{1}} \ldots\left(J^{\dagger}\right)_{b_{k}}^{j_{k}} \sigma_{L}^{I}\left(\sigma^{-1}\right)_{J}^{K} \\
& =\sum_{\rho \in S_{k}} \sum_{\sigma \in S_{n}} D_{a_{\rho(1)} b_{1}}^{H}(\sigma) \ldots D_{a_{\rho(k)} b_{k}}^{H}(\sigma) \sigma_{L}^{I}\left(\sigma^{-1}\right)_{J}^{K}
\end{aligned}
$$

Now notice that $A_{a_{1}}^{\dagger} A_{a_{2}}^{\dagger} \ldots A_{a_{k}}^{\dagger}$ is totally symmetric in the $a_{i}$ indices and thus lies in the totally symmetric tensor product $\operatorname{Sym}\left(V_{H}^{\otimes k}\right)$. Equivalently this is the projection in

$$
V_{H}^{\otimes k}=\bigoplus_{\Lambda_{1}, \Lambda_{2}} V_{\Lambda_{1}}^{\left(S_{n}\right)} \otimes V_{\Lambda_{2}}^{\left(S_{k}\right)} \otimes V_{\Lambda_{1}, \Lambda_{2}}
$$


to $\Lambda_{2}=[k]$. We can consider the corresponding Clebsch-Gordon coefficients to the irreducible representation $\Lambda_{1}$.

$$
C_{a_{1} \ldots a_{k}}^{\Lambda_{1},[k], m_{\Lambda_{1}} ; \tau}
$$

The index $\tau$ runs through the multiplicity of the irrep. $\Lambda_{1} \otimes[k]$ of $S_{n} \times S_{k}$ in $V_{H}^{\otimes k}$ which is the dimension of $V_{\Lambda,[k]}$. Equivalently, this is the multiplicity of $\Lambda_{1}$ of $S_{n}$ in $\operatorname{Sym}\left(V_{H}^{\otimes k}\right)$. Formulae for the multiplicities are given in Section 3. The Clebsch-Gordan coefficients are explained in Appendix Section A.

The CG coefficients have the following properties. Firstly

$$
C_{a_{1} \ldots a_{k}}^{\Lambda_{1},[k], m_{\Lambda_{1}} ; \tau}=C_{a_{\rho(1)} \ldots a_{\rho(k)}}^{\Lambda_{1},[k], m_{\Lambda_{1}} ; \tau} \quad \forall \rho \in S_{k}
$$

which simply reflects the fact that the $\mathrm{CG}$ coefficient couples to the symmetric product $\operatorname{Sym}\left(V_{H}^{\otimes k}\right)$. Secondly

$$
C_{a_{1} \ldots a_{k}}^{\Lambda_{1},[k], m_{\Lambda_{1}} ; \tau} D_{a_{1} b_{1}}^{H}(\sigma) \ldots D_{a_{k} b_{k}}^{H}(\sigma)=D_{m_{\Lambda_{1}} m_{\Lambda_{1}}^{\prime}}^{\Lambda_{1}}(\sigma) C_{b_{1} \ldots b_{k}}^{\Lambda_{1},[k], m_{\Lambda_{1}}^{\prime} ; \tau}
$$

which is derived in (160). Finally orthogonality:

$$
C_{a_{1} \ldots a_{k}}^{\Lambda_{1},[k], m_{\Lambda_{1}} ; \tau}\left(C^{\dagger}\right)_{a_{1} \ldots a_{k}}^{\Lambda_{1}^{\prime},[k], m_{\Lambda_{1}^{\prime}}^{\prime} ; \tau^{\prime}}=\delta_{\Lambda_{1} \Lambda_{1}^{\prime}} \delta_{m_{\Lambda_{1}} m_{\Lambda_{1}}^{\prime}} \delta_{\tau \tau^{\prime}}
$$

Now define new operators transforming in the irreps. of $S_{n}$

$$
\left(\mathcal{O}^{n, k ; \Lambda_{1}, m_{\Lambda_{1}} ; \tau}\right)_{J}^{I}=C_{a_{1} \ldots a_{k}}^{\Lambda_{1},[k], m_{\Lambda_{1}} ; \tau} \rho_{J}^{I}\left(A_{a_{1}}^{\dagger} \ldots A_{a_{k}}^{\dagger}|0\rangle\right)
$$

Using the properties of the Clebsch-Gordon coefficients we show that the operators $\mathcal{O}^{n, k ; \Lambda_{1}, m_{\Lambda_{1}} ; \tau_{\Lambda}}$ have the following correlation function

$$
\begin{aligned}
& \left\langle\left(\mathcal{O}^{n, k ; \Lambda_{1}, m_{\Lambda_{1}} ; \tau}\right)_{J}^{I}\left(\mathcal{O}^{\left.\dagger^{n^{\prime}, k^{\prime} ; \Lambda_{1}^{\prime}, m_{\Lambda_{1}^{\prime}}^{\prime} ; \tau^{\prime}}\right)}\right)_{L}^{K}\right\rangle \\
& =\delta^{n n^{\prime}} \delta^{k k^{\prime}} C_{a_{1} \ldots a_{k}}^{\Lambda_{1},[k], m_{\Lambda_{1}} ; \tau}\left(C^{\dagger}\right)_{b_{1} \ldots b_{k}}^{\Lambda_{1}^{\prime},[k], m_{\Lambda_{1}^{\prime}}^{\prime} ; \tau^{\prime}} \\
& \sum_{\rho \in S_{k}} \sum_{\sigma \in S_{n}} D_{a_{\rho(1)} b_{1}}^{H}(\sigma) \ldots D_{a_{\rho(k)} b_{k}}^{H}(\sigma) \sigma_{L}^{I}\left(\sigma^{-1}\right)_{J}^{K} \\
& =\delta^{n n^{\prime}} \delta^{k k^{\prime}} k ! C_{b_{1} \ldots b_{k}}^{\Lambda_{1},[k], \hat{m}_{\Lambda_{1}} ; \tau}\left(C^{\dagger}\right)_{b_{1} \ldots b_{k}}^{\Lambda_{1}^{\prime},[k], m_{\Lambda_{1}^{\prime}}^{\prime} ; \tau^{\prime}} \sum_{\sigma \in S_{n}} D_{m_{\Lambda_{1}} \hat{m}_{\Lambda_{1}}}^{\Lambda_{1}}(\sigma) \sigma_{L}^{I}\left(\sigma^{-1}\right)_{J}^{K} \\
& =\delta^{n n^{\prime}} \delta^{k k^{\prime}} \delta^{\Lambda_{1} \Lambda_{1}^{\prime}} \delta^{\tau \tau^{\prime}} k ! \sum_{\sigma \in S_{n}} D_{m_{\Lambda_{1}} m_{\Lambda_{1}}^{\prime}}^{\Lambda_{1}}(\sigma) \sigma_{L}^{I}\left(\sigma^{-1}\right)_{J}^{K}
\end{aligned}
$$

\subsection{Descendants}

We have a raising operator $\mathbf{L}_{+}$(see equation (16)) corresponding to a space-time derivative. Acting on the lowest weight state we obtain the descendant operator

$$
\mathcal{O}^{\Lambda=n+k, M_{\Lambda} ; \Lambda_{1}, m_{\Lambda_{1}} ; \tau}=\left(\mathbf{L}_{+}\right)^{M_{\Lambda}} C_{a_{1} \ldots a_{k}}^{\Lambda_{1},[k], m_{\Lambda_{1}} ; \tau} A_{a_{1}}^{\dagger} \ldots A_{a_{k}}^{\dagger}\left|\mathbf{X}_{J}^{I}\right\rangle
$$


We then find the expected canonical form, using the commutator relations in Section 2.1.

$$
\begin{aligned}
& \left\langle\mathcal{O}^{\Lambda=n+k, M_{\Lambda} ; \Lambda_{1}, m_{\Lambda_{1}} ; \tau}\left(\mathcal{O}^{\dagger}\right)^{\Lambda^{\prime}=n^{\prime}+k^{\prime}, M_{\Lambda^{\prime}}^{\prime} ; \Lambda_{1}^{\prime}, m_{\Lambda_{1}^{\prime}}^{\prime} ; \tau^{\prime}}\right\rangle \\
& =\delta^{n n^{\prime}} \delta^{k k^{\prime}} \delta^{M_{\Lambda} M_{\Lambda^{\prime}}^{\prime}} \delta^{\Lambda_{1} \Lambda_{1}^{\prime}} \delta^{\tau \tau^{\prime}}\left(M_{\Lambda} !\right)^{2} k ! \sum_{\sigma \in S_{n}} D_{m_{\Lambda_{1}} m_{\Lambda_{1}}^{\Lambda_{1}}}^{\Lambda_{1}}(\sigma) \sigma_{L}^{I}\left(\sigma^{-1}\right)_{J}^{K}
\end{aligned}
$$

\section{Multiplicities of $S L(2) \times S_{n}$ irreps. in $V_{F}^{\otimes n}$}

In this section we give formulae for the multiplicities of the operators found in (37) using characters.

\subsection{Multiplicity of $S L(2)$ irreps.}

We begin by considering the multiplicities of $S L(2)$ irreps in $V_{F}^{\otimes n}$ which includes a sum over $S_{n}$ irreps. The states $\partial^{l} X$ in $V_{F}$ have weights $L_{0}=1+l$, with $l$ going up to infinity. They form a lowest weight discrete series irrep $V_{1}=V_{F}$. Similar discrete series irreps exist for any $k$, i.e. $V_{k}$. We wish to find the tensor product decomposition of $V_{1}^{\otimes n}$ in terms of the irreps. $V_{k}$. This can be derived by characters. The character of the irrep. $V_{k}$ is

$$
\chi_{k}(q):=\operatorname{Tr}_{V_{k}}\left(q^{L_{0}}\right)=q^{k} \sum_{l=0}^{\infty} q^{l}=\frac{q^{k}}{(1-q)}
$$

For the tensor product $V_{1}^{\otimes n}$ we get the character

$$
\begin{aligned}
\left(\chi_{1}(q)\right)^{n} & =\frac{q^{n}}{(1-q)} \frac{1}{(1-q)^{n-1}} \\
& =\frac{q^{n}}{(1-q)} \sum_{k \geq 0} \frac{(n-2+k) !}{k !(n-2) !} q^{k} \\
& =\sum_{k \geq 0} \chi_{n+k}(q) m(k, n)
\end{aligned}
$$

where we have defined

$$
m(k, n)=\frac{(n-2+k) !}{k !(n-2) !}
$$

We thus have the decomposition of $V_{F}^{\otimes n}$ as:

$$
V_{F}^{\otimes n}=\bigoplus_{k \geq 0} m(k, n) \quad V_{n+k}
$$

We have seen in the previous section that the multiplicity $m(k, n)$ is generated by $k$ powers of the oscillators $A_{a}^{\dagger}$. They transform in the $H=[n-1,1]$ representation of $S_{n}$ with dimension $(n-1)$. The $k$-oscillator states transform in the symmetrised tensor product of 
the hook representation which does indeed have dimension $m(k, n)$. The multiplicity $m(k, n)$ can be decomposed into irreps of $S_{n}$ by finding the decomposition of $\operatorname{Sym}\left(V_{H}^{\otimes k}\right)$ into irreps of $S_{n}$. We can therefore write

$$
\begin{aligned}
V_{1}^{\otimes n} & =\bigoplus_{k} V_{\Lambda=n+k} \otimes \operatorname{Sym}\left(V_{H}^{\otimes k}\right) \\
& =\bigoplus_{k} V_{\Lambda=n+k}^{S L(2)} \otimes V_{\Lambda_{1}}^{S_{n}} \otimes V_{\Lambda, \Lambda_{1}}^{\operatorname{Com}\left(S L(2) \times S_{n}\right)}
\end{aligned}
$$

Here the integer $k$ runs from 0 to infinity and $\operatorname{Com}\left(S L(2) \times S_{n}\right)$ is the commutant of $S L(2) \times$ $S_{n}$. Now if we act with a projector $P_{\Lambda_{1}}=\frac{d_{\Lambda_{1}}}{n !} \sum_{\sigma} \chi_{\Lambda_{1}}(\sigma) \sigma$ on $V_{1}^{\otimes n}$ we will project out the subspace with a fixed $\Lambda_{1}$. Taking the $S L(2)$ character in this $\Lambda_{1}$-symmetrised subspace and expanding in terms of the characters of $V_{p}$ will yield the dimensions of the multiplicity spaces $V_{\Lambda, \Lambda_{1}}^{\operatorname{Com}\left(S L(2) \times S_{n}\right)}$. This provides a way to solve the problem of $\operatorname{decomposing} \operatorname{Sym}\left(V_{H}^{\otimes k}\right)$ into irreps of $S_{n}$. We consider this problem in the next section.

\subsection{Multiplicities of $S L(2) \times S_{n}$ and $q$-deformed $G L(\infty)$}

\subsubsection{Examples of symmetric and antisymmetric $S_{n}$ irreps}

As an example of this method, take $\Lambda_{1}=[n]$ the symmetric irrep. We want to calculate $\operatorname{tr}_{\mathcal{W}} P_{\Lambda_{1}} q^{L_{0}}$ where the trace is taken over $\mathcal{W}=V_{1}^{\otimes n}$. This means calculating $q^{L_{0}}$ in the symmetrised subspace of $V_{1}^{\otimes n}$. A basis in the symmetrised subspace of $\left|m_{1}, m_{2}, . ., m_{n}\right\rangle$ is in $1-1$ correspondence with natural numbers $m_{1}, m_{2}, \cdots m_{n}$ obeying

$$
0 \leq m_{1} \leq m_{2} \leq \cdots m_{n} \leq \infty
$$

So the character is

$$
\begin{aligned}
\operatorname{tr}_{\mathcal{W}} P_{[n]} q^{L_{0}} & =q^{n} \sum_{m_{n}=0}^{\infty} \sum_{m_{n-1}=0}^{m_{n}} \cdots \sum_{m_{2}=0}^{m_{3}} \sum_{m_{1}=0}^{m_{2}} q^{m_{1}+m_{2}+\cdots+m_{n}} \\
& =q^{n} \prod_{i=1}^{n} \frac{1}{\left(1-q^{i}\right)} \\
& =\frac{q^{n}}{(1-q)} \prod_{i=2}^{n} \frac{1}{\left(1-q^{i}\right)}
\end{aligned}
$$

The multiplicity of $V_{\Lambda=n+k}^{S L(2)} \otimes V_{[n]}^{\left(S_{n}\right)}$ is then the coefficient of $q^{k}$ in the generating function

$$
\prod_{i=2}^{n} \frac{1}{\left(1-q^{i}\right)}
$$

As an example for $n=2$, the multiplicity of $V_{2+k}$ is the coefficient of $q^{k}$ in $\frac{1}{1-q^{2}}$. This tells us that the symmetric irrep. of $S_{n}$ only appears for $k=0,2,4, \cdots$ with unit multiplicity. 
Similarly, for $R=\left[1^{n}\right]$ we apply the antisymmetric projector to $\mathcal{W}$ we have a basis in correspondence with $\left(m_{1}, m_{2}, \cdots, m_{n}\right)$ with $m_{1}<m_{2}<\cdots<m_{n}$. So the character is

$$
\begin{aligned}
\operatorname{tr}_{\mathcal{W}}\left(P_{\left[1^{n}\right]} q^{L_{0}}\right) & =q^{n} \sum_{m_{n}=n-1}^{\infty} \sum_{m_{n-1}=n-2}^{m_{n-1}-1} \cdots \sum_{m_{2}=1}^{n} \sum_{m_{1}=0}^{m_{3}-1} q^{m_{1}+m_{2}+\cdots+m_{n}} \\
& =q^{n} q^{\frac{n(n-1)}{2}} \prod_{i=1}^{n} \frac{1}{1-q^{i}} \\
& =\frac{q^{n}}{1-q} q^{\frac{n(n-1)}{2}} \prod_{i=2}^{n} \frac{1}{1-q^{i}}
\end{aligned}
$$

So the number of antisymmetric [1 $1^{n}$ irreps. of $S_{n}$ in the multiplicity space of $V_{n+k}$ is the coefficient of $q^{k}$ in

$$
\frac{q^{\frac{n(n-1)}{2}}}{\left(1-q^{2}\right) \cdots\left(1-q^{n}\right)}
$$

This multiplicity is zero unless $k \geq \frac{n(n-1)}{2}$. This is as it should be because the antisymmetry condition means that we need $X, \partial X, \ldots \partial^{n-1} X$ which has weight $n+\frac{n(n-1)}{2}$.

\subsubsection{The generating function for any $S L(2) \times S_{n}$ irreps}

In fact it turns out we can write down a compact formula for the generating function for the multiplicities of $V_{\Lambda=n+k} \otimes V_{\Lambda_{1}}$ in $\mathcal{W}$ for any $\Lambda_{1}$. It is given by

$$
(1-q) q^{\sum_{i=1} \frac{c_{i}\left(c_{i}-1\right)}{2}} \prod_{b} \frac{1}{\left(1-q^{h_{b}}\right)}
$$

The product runs over the boxes of the Young diagram of $\Lambda_{1}$ and $h_{b}$ is the hook length of the box. $c_{i}$ is the column length of the $i$ 'th column. One can check that this agrees with (48) and (50) for $R=[n]$ and $R=\left[1^{n}\right]$.

The proof of this generating function, using $q$-dimensions of $G L(\infty)$, goes as follows.

\subsubsection{Proof using $q$-dimensions of $G L(\infty)$}

It is useful to think of the infinite dimensional representation $V_{F}$ as a limit of finite dimensional representations $V_{\tilde{N}}$ of $G L(\tilde{N})$ for $\tilde{N} \rightarrow \infty$. This corresponds to considering fundamental fields of the form $\partial^{k} X$ for $0 \leq k \leq \tilde{N}-1$. States in $V_{F}^{\otimes n}=V_{\tilde{N}}^{\otimes n}$ of a fixed $S_{n}$ symmetry given by Young diagram $\Lambda_{1}$, can be labelled by inserting $n$ positive integers from $1 \cdots \tilde{N}$ into the Young diagram, with the numbers strictly decreasing down the columns and weakly increasing along the rows. These are the semi-standard Young tableaux [29]. We will denote by $\vec{m}(R)$ a set of numbers corresponding to a semi-standard Young tableau. This corresponds to operators consisting of the letters $\partial^{m_{1}-1} X, \ldots, \partial^{m_{n}-1} X$. So we have

$$
\operatorname{tr}_{\mathcal{W}} P_{\Lambda_{1}} q^{L_{0}}=\sum_{\vec{m}(R)} q^{m_{1}+m_{2}+\cdots m_{n}}
$$


In a standard basis of $G L(\tilde{N})$, with $e_{i}$ being the column vector with 1 in the $i$ 'th place and 0 elsewhere, the diagonal matrices $E_{i i}$ act as

$$
E_{i i} e_{m}=\delta_{i m} e_{m}
$$

The weight $q^{m}$ can be identified with the eigenvalues of $q^{\sum_{m} m E_{m m}}$. Precisely this generator appears in the computation of the $q$-dimension of the representation $\Lambda_{1}$ of $U_{q}(G L(\tilde{N}))$. This sum also appears in studying the decomposition of $V_{j}^{\otimes n}$ for the $S U(2)$ representation $V_{j}$ $(2 j+1=\tilde{N})$ in terms of $S U(2) \times S_{n}[19]$. It is known to be

$$
q^{n+\sum_{i} \frac{c_{i}\left(c_{i}-1\right)}{2}} \prod_{i, j} \frac{\left(1-q^{\tilde{N}-i+j}\right)}{1-q^{h(i, j)}}
$$

where $i$ runs along the columns, $j$ runs along the rows of the Young diagram, and $h(i, j)$ is the hook length of the box labelled by $(i, j)$. When $\tilde{N} \rightarrow \infty$ in a region of $q<1$ ( where the desired sums converge), the numerator goes to 1 and we get the result

$$
\operatorname{tr}_{\mathcal{W}} P_{\Lambda_{1}} q^{L_{0}}=q^{n+\sum_{i} \frac{c_{i}\left(c_{i}-1\right)}{2}} \prod_{i, j} \frac{1}{1-q^{h(i, j)}}
$$

Factoring out the character of $V_{\Lambda=n+k}$ which is $\frac{q^{n+k}}{(1-q)}$ we get the multiplicity of this representation. This proves the claim that the coefficient of $q^{k}$ in (51) is the multiplicity of the representation $V_{\Lambda=n+k}$ in $\mathcal{W}$.

\subsection{The general case using characters of symmetric groups $S_{n} \times S_{k}$}

As we explained in Section 3.1, the oscillator construction of $S L(2)$ representations implies that the multiplicity of the $S L(2) \times S_{n}$ representation $V_{\Lambda=n+k} \otimes V_{\Lambda_{1}}$ in the decomposition of $V_{1}^{\otimes n}$ is given by the multiplicity of $V_{\Lambda_{1}}$ of $S_{n}$ in $\operatorname{Sym}\left(V_{H}^{\otimes k}\right)$. Equivalently this is the multiplicity of the representation $\Lambda_{1} \otimes[k]$ of $S_{n} \times S_{k}$ in $V_{H}^{\otimes k}$, where $[k]$ denotes the Young diagram of $S_{k}$ with a single row of length $k$ which is the symmetric representation. The projectors $P_{\Lambda_{1}} \otimes P_{[k]}$ can be written down using characters of symmetric groups. Hence we have

$$
\begin{aligned}
d_{\Lambda=n+k, \Lambda_{1}} & =\operatorname{tr}_{V_{H}}\left(P_{\Lambda_{1}} \otimes P_{[k]}\right) \\
& =\frac{1}{n !} \sum_{\sigma \in S_{n}} \chi_{\Lambda_{1}}(\sigma) \frac{1}{k !} \sum_{\tau \in S_{k}} \chi_{[k]}(\tau) \prod_{i}\left(\operatorname{tr}_{V_{H}}\left(\sigma^{i}\right)\right)^{c_{i}(\tau)}
\end{aligned}
$$

$c_{i}(\tau)$ is the number of cycles in $\tau$ of length $i$. See Appendix Section A.1 for further details. 
We can check that this multiplicity gives the correct $m(k, n)$

$$
\begin{aligned}
m(k, n) & =\sum_{\Lambda_{1}\left(S_{n}\right)} d_{\Lambda_{1}} d_{\Lambda=n+k, \Lambda_{1}} \\
& =\sum_{\sigma \in S_{n}} \sum_{\Lambda_{1}\left(S_{n}\right)} \frac{1}{n !} d_{\Lambda_{1}} \chi_{\Lambda_{1}}(\sigma) \frac{1}{k !} \sum_{\tau \in S_{k}} \prod_{i}\left(\operatorname{tr}_{V_{H}}\left(\sigma^{i}\right)\right)^{c_{i}(\tau)} \\
& =\frac{1}{k !} \sum_{\tau \in S_{k}}\left(\operatorname{tr}_{V_{H}}(\mathrm{id})\right)^{\sum_{i} c_{i}(\tau)} \\
& =\operatorname{dim}_{n-1}[k] \\
& =\frac{(n-2+k) !}{k !(n-2) !}
\end{aligned}
$$

We have identified $m(k, n)$ with the dimension of the totally symmetric $G L(n-1)$ representation $[k]$.

\section{General $G, V$}

We now consider how the derivation of the canonical covariant form, such as (38) in the $S L(2)$ case, generalises to the situation of any global symmetry group $G$. The Lie algebra generators act on $n$-fold tensor products of representations $V_{1} \otimes V_{2} \cdots \otimes V_{n}$ as

$$
\Delta_{n}\left(J_{a}\right)=J_{a} \otimes 1 \otimes \cdots \otimes 1+1 \otimes J_{a} \otimes \cdots \otimes 1+\ldots+1 \otimes \cdots \otimes J_{a}
$$

In particular we will be interested in the $n$-fold tensor product of the representation $V_{F}$ corresponding to the fundamental fields in (a sector of) the theory. Note that the action of $G$ commutes with the symmetric group action permuting the $n$ factors in the tensor product. For any $\sigma \in S_{n}$ we have in $\operatorname{End}\left(V_{F}^{\otimes n}\right)$

$$
\sigma \Delta_{n}\left(J_{a}\right)=\Delta_{n}\left(J_{a}\right) \sigma
$$

The signs that arise in the super-algebra case will be discussed in section 4.1.1, where we will show that the key result generalizes.

We will now organise the states in $V_{F}^{\otimes n}$ according to the representations of the product group $G \times S_{n}$ acting on this space

$$
\left(V_{F}^{G}\right)^{\otimes n}=\oplus_{\Lambda, \Lambda_{1}} V_{\Lambda}^{G} \otimes V_{\Lambda_{1}}^{S_{n}} \otimes V_{\Lambda, \Lambda_{1}}^{\mathrm{Com}\left(G \times S_{n}\right)}
$$

The $n$-fold tensor product has states

$$
\left|m_{1}, m_{2}, \cdots, m_{n}\right\rangle
$$

where $m$ runs over the states in the fundamental representation (which can be infinite dimensional as in the $S L(2)$ case). We can choose an orthonormal basis

$$
\left\langle m_{1}, m_{2}, \cdots m_{n} \mid m_{1}^{\prime}, m_{2}^{\prime}, \cdots, m_{n}^{\prime}\right\rangle=\delta_{m_{1} m_{1}^{\prime}} \cdots \delta_{m_{n} m_{n}^{\prime}}
$$


We can decompose the $n$-fold tensor product into irreps of the global symmetry as follows

$$
\left|m_{1}, \cdots, m_{n}\right\rangle=\sum_{\Lambda, M_{\Lambda}, i} C_{\vec{m}}^{\Lambda, M_{\Lambda}, i}\left|\Lambda, M_{\Lambda}, i\right\rangle
$$

$M_{\Lambda}$ is the state within $V_{\Lambda}$ and $i$ is a multiplicity index for $V_{\Lambda}$. In fact $V^{\otimes n}$ has an action of $G \times S_{n}$ so we can decompose the above multiplicity index $i$ into $\left(\Lambda_{1}, m_{\Lambda_{1}}, \tau\right)$ where $\Lambda_{1}$ labels an $S_{n}$ irrep, $m_{\Lambda_{1}}$ runs over the states in the irrep $\Lambda_{1}$ of $S_{n}$ and $\tau$ labels the multiplicity of $V_{\Lambda} \otimes V_{\Lambda_{1}}$. We define the Clebsch for this decomposition

$$
C_{\vec{m}}^{\Lambda, M_{\Lambda}, \Lambda_{1}, m_{\Lambda_{1}}, \tau}=\left\langle\Lambda, M_{\Lambda}, \Lambda_{1}, m_{\Lambda_{1}}, \tau \mid \vec{m}\right\rangle
$$

The Clebsch are invertible

$$
\left|\Lambda, M_{\Lambda}, \Lambda_{1}, m_{\Lambda_{1}}, \tau\right\rangle=\sum_{\vec{m}} C_{\Lambda, M_{\Lambda}, \Lambda_{1}, m_{\Lambda_{1}}, \tau}^{\vec{m}}|\vec{m}\rangle
$$

so that

$$
C_{\Lambda, M_{\Lambda}, \Lambda_{1}, m_{\Lambda_{1}}, \tau}^{\vec{m}}=\left\langle\vec{m} \mid \Lambda, M_{\Lambda}, \Lambda_{1}, m_{\Lambda_{1}}, \tau\right\rangle
$$

Using the hermiticity of the inner product

$$
C_{\Lambda, M_{\Lambda}, \Lambda_{1}, m_{\Lambda_{1}}, \tau}^{\vec{m}}=\left(C_{\vec{m}}^{\Lambda, M_{\Lambda}, \Lambda_{1}, m_{\Lambda_{1}}, \tau}\right)^{*}
$$

We can always choose an orthonormal basis

$$
\left\langle\Lambda, M_{\Lambda}, \Lambda_{1}, m_{\Lambda_{1}}, \tau \mid \Lambda^{\prime}, M_{\Lambda^{\prime}}^{\prime}, \Lambda_{1}^{\prime}, m_{\Lambda_{1}^{\prime}}^{\prime}, \tau^{\prime}\right\rangle=\delta_{\Lambda \Lambda^{\prime}} \delta_{M_{\Lambda} M_{\Lambda^{\prime}}^{\prime}} \delta_{\Lambda_{1} \Lambda_{1}^{\prime}} \delta_{m_{\Lambda_{1}} m_{\Lambda_{1}^{\prime}}^{\prime}} \delta_{\tau \tau^{\prime}}
$$

which leads to orthogonality of the Clebsch

$$
\sum_{\vec{m}}\left(C_{\vec{m}}^{\Lambda, M_{\Lambda}, \Lambda_{1}, m_{\Lambda_{1}}, \tau}\right)^{*} C_{\vec{m}}^{\Lambda^{\prime}, M_{\Lambda^{\prime}}^{\prime}, \Lambda_{1}^{\prime}, m_{\Lambda_{1}^{\prime}}^{\prime}, \tau^{\prime}}=\delta_{\Lambda \Lambda^{\prime}} \delta_{M_{\Lambda} M_{\Lambda^{\prime}}^{\prime}} \delta_{\Lambda_{1} \Lambda_{1}^{\prime}} \delta_{m_{\Lambda_{1}} m_{\Lambda_{1}^{\prime}}^{\prime}} \delta_{\tau \tau^{\prime}}
$$

\subsection{Correlators in free field theory}

Corresponding to the orthogonal states in $V_{F}$ we have fields $W_{m}$ which diagonalize the Zamolodchikov metric

$$
\left\langle W_{m} W_{m^{\prime}}\right\rangle=\delta_{m m^{\prime}}
$$

For the states in $V_{F}^{\otimes n}$ take operators $\mathcal{O}_{m_{1}, m_{2}, \cdots m_{n}} \equiv \mathcal{O}_{\vec{m}}=W_{m_{1}} \otimes W_{m_{2}} \cdots W_{m_{n}}$. In the case of $S L(2)$ this is

$$
\frac{1}{m_{1} ! m_{2} ! \cdots m_{n} !} \partial^{m_{1}} X \otimes \partial^{m_{2}} X \cdots \otimes \partial^{m_{n}} X
$$


The 2-point function can be written as

$$
\left\langle\left(\mathcal{O}_{m_{1}, m_{2}, \cdots m_{n}}^{\dagger}\right)_{J}^{I},\left(\mathcal{O}_{m_{1}^{\prime}, m_{2}^{\prime} \cdots m_{n}^{\prime}}\right)_{L}^{K}\right\rangle=\sum_{\sigma \in S_{n}} \prod_{i=1}^{n} \delta_{m_{i} m_{\sigma(i)}^{\prime}}(\sigma)_{J}^{K}\left(\sigma^{-1}\right)_{L}^{I}
$$

where the sum over $\sigma$ runs over Wick contractions and we have used the orthogonality (70). Define operators in correspondence with the orthonormal $G \times S_{n}$ basis of the $V^{\otimes n}$ :

$$
\mathcal{O}_{\Lambda, M_{\Lambda}, \Lambda_{1}, m_{\Lambda_{1}, \tau}}=\sum_{\vec{m}} C_{\Lambda, M_{\Lambda}, \Lambda_{1}, m_{\Lambda_{1}}, \tau}^{\vec{m}} \mathcal{O}_{m_{1}, \cdots, m_{n}}
$$

The correlator of the gauge-covariant operators is

$$
\begin{aligned}
& \left\langle\left(\mathcal{O}_{\Lambda, M_{\Lambda}, \Lambda_{1}, m_{\Lambda_{1}}, \tau}^{\dagger}\right)_{J}^{I}\left(\mathcal{O}_{\Lambda^{\prime}, M_{\Lambda^{\prime}}^{\prime}, \Lambda_{1}^{\prime}, m_{\Lambda_{1}^{\prime}}^{\prime}, \tau^{\prime}}\right)_{L}^{K}\right\rangle \\
& =\sum_{\vec{m}, \vec{m}^{\prime}}\left(C_{\Lambda, M_{\Lambda}, \Lambda_{1}, m_{\Lambda_{1}}, \tau}^{m_{1} \cdots m_{n}}\right)^{*} C_{\Lambda^{\prime}, M_{\Lambda^{\prime}}^{\prime} \Lambda_{1}^{\prime}, m_{\Lambda_{1}^{\prime}}^{\prime}, \tau^{\prime}}^{m_{1}^{\prime} \cdots m_{\sigma \in S_{n}}^{\prime}} \sum_{i=1}^{n} \delta_{m_{i} m_{\sigma(i)}^{\prime}}(\sigma)_{J}^{K}\left(\sigma^{-1}\right)_{L}^{I}
\end{aligned}
$$

Solve the delta function

$$
\begin{aligned}
& \left\langle\left(\mathcal{O}_{\Lambda, M_{\Lambda}, \Lambda_{1}, m_{\Lambda_{1}, \tau}, \tau}^{\dagger}\right)_{J}^{I}\left(\mathcal{O}_{\Lambda^{\prime}, M_{\Lambda^{\prime}}^{\prime}, \Lambda_{1}^{\prime}, m_{\Lambda_{1}^{\prime}, \tau^{\prime}}^{\prime}}\right)_{L}^{K}\right\rangle \\
& =\sum_{\sigma \in S_{n}} \sum_{\vec{m}}\left(C_{\Lambda, M_{\Lambda}, \Lambda_{1}, m_{\Lambda_{1}}, \tau}^{m_{1} \cdots m_{n}}\right)^{*} C_{\Lambda^{\prime}, M_{\Lambda^{\prime}}^{\prime}, \Lambda_{1}^{\prime}, m_{\Lambda_{1}^{\prime}}^{\prime}, \tau^{\prime}}^{m_{\sigma^{-1}}(1) \cdots m_{\sigma^{-1}(n)}} \quad(\sigma)_{J}^{K}\left(\sigma^{-1}\right)_{L}^{I}
\end{aligned}
$$

We can simplify the $\sigma^{-1}$ action on $V_{F}^{\otimes n}$ in the second Clebsch because we know it transforms under the $S_{n}$ representation $\Lambda_{1}^{\prime}$ : the action is just the matrix representation of $\Lambda_{1}^{\prime}$.

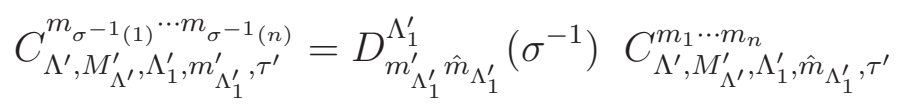

Use this and the orthogonality of the Clebsch from equation (69) to get

$$
\left\langle\left(\mathcal{O}_{\Lambda, M_{\Lambda}, \Lambda_{1}, m_{\Lambda_{1}}, \tau}^{\dagger}\right)_{J}^{I}\left(\mathcal{O}_{\Lambda^{\prime}, M_{\Lambda^{\prime}}^{\prime}, \Lambda_{1}^{\prime}, m_{\Lambda_{1}^{\prime}}^{\prime}, \tau^{\prime}}\right)_{L}^{K}\right\rangle=\delta_{\Lambda \Lambda^{\prime}} \delta_{M_{\Lambda} M_{\Lambda^{\prime}}^{\prime}} \delta_{\Lambda_{1} \Lambda_{1}^{\prime}} \delta_{\tau \tau^{\prime}} \sum_{\sigma \in S_{n}} D_{m_{\Lambda_{1}} m_{\Lambda_{1}^{\prime}}^{\prime}}^{\Lambda_{1}}(\sigma)(\sigma)_{J}^{K}\left(\sigma^{-1}\right)_{L}^{I}
$$

\subsubsection{Signs in the super-algebra case}

In the case where the generators of $G$ include fermionic ones, there is a small modification of the above proof. Fermionic generators $Q$ pick up signs when taken past fermionic fields

$$
Q\left(\psi_{1} \psi_{2}\right)=\left(Q \psi_{1}\right) \psi_{2}-\psi_{1}\left(Q \psi_{2}\right)
$$

In this case the action of permutations is defined with a sign for each fermion swap. For the transposition of $\psi_{1} \psi_{2}$ we define

$$
s\left(\psi_{1} \psi_{2}\right)=-\psi_{2} \psi_{1}
$$


It is easy to check that $Q s=s Q$. This is the key point. If we define the action of permutations to pick up a sign for every swap of fermions, we have an action of the permutation group which commutes with the super-algebra. Hence Clebsch-Gordan coefficients for $G \times S_{n}$ are well-defined, and we can define operators according to (73). The two-point function in this case picks up a sign $(-1)^{\epsilon(\vec{m}, \sigma)}$ on the RHS of (74). This sign carries into (75). Because the correct action of the permutations involves this same sign factor, equation (76) has the sign $(-1)^{\epsilon(\vec{m}, \sigma)}$ on the left. The final result (177) remains unchanged.

\subsection{Completeness}

The completeness of these operators in $V_{F}^{\otimes n}$ follows from the invertibility of the Clebschs; this means that any state in $V_{F}^{\otimes n}$ can be written as a linear combination of states in $V_{\Lambda} \otimes V_{\Lambda_{1}}$.

$$
\mathcal{O}_{m_{1}, \cdots m_{n}}=\sum_{\Lambda, M_{\Lambda}, \Lambda_{1}, m_{\Lambda_{1}}, \tau} C_{\vec{m}}^{\Lambda, M_{\Lambda}, \Lambda_{1}, m_{\Lambda_{1}}, \tau} \mathcal{O}_{\Lambda, M_{\Lambda}, \Lambda_{1}, m_{\Lambda_{1}}, \tau}
$$

If we reintroduce the gauge indices and trace our covariant operators to get gaugeinvariant operators, then we must also take into account finite $N$ constraints. This is studied in Section 6.

\section{$5 \quad$ Examples and applications}

The above formalism can be applied to any global symmetry $G$ of a theory, or to a subgroup of the global symmetry acting on a sector of the fields. In this section we will consider a number of examples to which we can apply this formalism. Examples relating to $\mathcal{N}=4 \mathrm{SYM}$ are the $S L(2)$ sector described above and the $U(M)$ (BPS) sector considered in [15]. We will review both of these sectors from the new perspective of the previous section. Free conformal field theories also possess an enlarged symmetry group known as higher spin symmetry and we will also see in this section how our construction naturally assembles into representations of this.

Another sector we will consider corresponds to the 6 hermitian scalars of $\mathcal{N}=4 \mathrm{SYM}$ and the $S O(6)$ subgroup of the global symmetry acting on them. The problem of diagonalising the gauge invariant operators amounts to first finding a manageable form of the ClebschGordan coefficients for the $S O(6) \times S_{n}$ decomposition of the $n$-fold tensor product of the fundamental of $S O(6)$. We will not solve this problem explicitly but will note some facts about the relevant multiplicities in Section 5.4. A more explicit description of the ClebschGordans analogous to what we gave for $U(M)$ in Section 5.2 would be desirable.

The sector of six scalars can also be described in terms of the 3 complex scalars and their conjugates. In this case, it is natural to use the $U(3)$ subgroup of $S O(6)$. The case of purely holomorphic operators was solved in [15] and is reviewed in 5.2. It should be possible to include the anti-holomorphic operators by using $U(3)$ along with the Brauer algebra $B_{N}(m, n)$ used in [14] for the case of a single complex scalar. 
Finally we will look at the formalism applied to product groups. To handle a class of derivatives of the holomorphic scalars we can use the product group technology applied to $S L(2) \times U(3)$. A simple sector including one chiral fermion, 3 holomorphic scalars is controlled by $S L(2) \times U(3 \mid 1)$. For the six hermitian scalars and a class of their derivatives we can use $S L(2) \times S O(6)$. For more general derivatives we can use $S O(4,2) \times S O(6)$. For the sixteenth BPS sector we would use $S U(3 \mid 2,1)$ [34]. For the complete set of fields of $\mathcal{N}=4$ SYM we can use the full symmetry $S U(2,2 \mid 4)$. Calculating the Clebsch-Gordan coefficients for the $V_{F}^{\otimes n}$ will allow, following the derivation of (77) from (69), to get the canonical covariant form which in turn leads to diagonal gauge invariant operators using Section 6 , The relevant Clebsch-multiplicities are known for $n=2$ [26]. Finding the multiplicities and the Clebsch-coefficients in terms of symmetric groups, as we do below for $U(M)$ and $S L(2)$, is the next step in the solution of the free field diagonalisation problem for $\mathcal{N}=4 \mathrm{SYM}$.

\section{$5.1 S L(2)$ and $\operatorname{Sym}\left(V_{H}^{\otimes k}\right)$}

Here we illustrate our scheme with the construction of the Clebsch $C_{\Lambda, M_{\Lambda}, \Lambda_{1}, m_{\Lambda_{1}}, \tau}^{\vec{m}}$ for the specific example of $G=S L(2)$, which we considered in section 2.

In the Fock space of oscillators we have

$$
\left|m_{1}, \cdots, m_{n}\right\rangle \sim\left(a_{1}^{\dagger}\right)^{m_{1}} \ldots\left(a_{n}^{\dagger}\right)^{m_{n}}|0\rangle
$$

whereas the oscillators $A_{a}$ give the decomposition in terms of $S L(2)$ irreps $\Lambda=n+k$, following equation (63)

$$
\left|\Lambda, M ;\left(a_{1}, \ldots a_{k}\right)\right\rangle=C_{\vec{m}}^{\Lambda, M,\left(a_{1} \ldots a_{k}\right)}\left|m_{1}, \cdots, m_{n}\right\rangle=A_{\left(a_{1}\right.}^{\dagger} \ldots A_{\left.a_{k}\right)}^{\dagger}\left(\mathbf{L}_{+}\right)^{M}|0\rangle
$$

so the label $i$ in equation (63) is given by the indices $\left(a_{1}, \ldots a_{k}\right)$. The Clebsch-Gordon coefficients of equation (63) can now be read off.

The next step is to decompose the label $i$ into irreps of $S_{n}$. The indices $a_{i}$ carry the $n-1$ dimensional hook representation, $H=[n-1,1]$ of $S_{n}$. Therefore the label $i=$ $\left(a_{1} \ldots a_{k}\right)$ carries the reducible representation $\operatorname{Sym} V_{H}^{\otimes k}$. This decomposes into the irreducible representations $\Lambda_{1}$ of $S_{n}$ with multiplicity $\tau$ via the Clebsch Gordon coefficient $C_{\left(a_{1} \ldots a_{k}\right)}^{\Lambda_{1}, m_{\Lambda_{1}}, \tau}$

$$
\left|\left(a_{1} \ldots a_{k}\right)\right\rangle=C_{\Lambda_{1}, m_{\Lambda_{1}, \tau}}^{\left(a_{1} \ldots a_{k}\right)}\left|\Lambda_{1}, m_{\Lambda_{1}}, \tau\right\rangle
$$

and the inverse transformation is given by $C_{\Lambda_{1}, m_{\Lambda_{1}}, \tau}^{\left(a_{1} \ldots a_{k}\right)}$ as

$$
\left|\Lambda_{1}, m_{\Lambda_{1}}, \tau\right\rangle=C_{\left(a_{1} \ldots a_{k}\right)}^{\Lambda_{1}, m_{\Lambda_{1}}, \tau}\left|\left(a_{1} \ldots a_{k}\right)\right\rangle
$$

Putting all this together we get

$$
\begin{aligned}
\left|\Lambda, M, \Lambda_{1}, m_{\Lambda_{1}}, \tau\right\rangle & =C_{\left(a_{1} \ldots a_{k}\right)}^{\Lambda_{1}, m_{\Lambda_{1}}, \tau}\left|\Lambda, M ;\left(a_{1}, \ldots a_{n}\right)\right\rangle \\
& =C_{\left(a_{1} \ldots a_{k}\right)}^{\Lambda_{1}, m_{\Lambda_{1}}, \tau} C_{\vec{m}}^{\Lambda, M,\left(a_{1} \ldots a_{k}\right)}\left|m_{1}, \cdots, m_{n}\right\rangle
\end{aligned}
$$

The Clebsch of equation (65) is given by

$$
C_{\vec{m}}^{\Lambda, M, \Lambda_{1}, m_{\Lambda_{1}}, \tau}=C_{\left(a_{1} \ldots a_{k}\right)}^{\Lambda_{1}, m_{\Lambda_{1}}, \tau} C_{\vec{m}}^{\Lambda, M,\left(a_{1} \ldots a_{k}\right)}
$$




\section{$5.2 U(M)$ revisited}

This formalism also applies to the $U(M)$ case studied in [15]. In that case the starting point is

$$
\left|m_{1}, \cdots, m_{n}\right\rangle \sim X_{m_{1}} \otimes \cdots \otimes X_{m_{n}} \quad m_{i}=1 \ldots M
$$

which is the tensor product of $n$ fundamentals of $U(M), V_{M}^{\otimes n}$. Then in (63) $C_{\vec{m}}^{\Lambda, M, i}$ is the $U(M)$ Clebsch Gordon coefficient decomposing $V_{M}^{\otimes n}$ into irreps $\Lambda$ with multiplicity $i$. In this case Schur Weyl duality tells us that $i$ also carries the fundamental of the representation $\Lambda$ of $S_{n}$. Thus $\Lambda_{1}=\Lambda$ and there is no $\tau$ multiplicity.

To be explicit, to get the operators of [15]

$$
C_{\Lambda, M, \Lambda_{1}=\Lambda, m_{\Lambda_{1}}=i}^{\vec{m}}=\frac{1}{n !} \sum_{\sigma \in S_{n}} B_{j \beta} D_{i j}^{\Lambda}(\sigma) \prod_{k=1}^{n} \delta_{m_{k} p_{\sigma^{-1}(k)}}
$$

Here $M=(\mu, \beta) . \mu$ labels the number of different flavour fields in the operator $\left(\mu_{1} X^{\prime}\right.$ 's, $\mu_{2}$ $Y$ 's, etc.), while $\beta$ runs over the number times the trivial representation of $H_{\mu}=S_{\mu_{1}} \times \cdots S_{\mu_{M}}$ is contained in $S_{n} . B_{j \beta}$ is a branching coefficient for the change of basis for the subspace of the irrep. $\Lambda$ invariant under $H_{\mu}$. Canonically we choose $p_{1}, \ldots p_{\mu_{1}}=1, p_{\mu_{1}+1}, \ldots p_{\mu_{1}+\mu_{2}}=2$, .... With this choice we recover the covariant operators in [15]

$$
\mathcal{O}_{i \beta}^{\Lambda \mu}=\sum_{\vec{m}} C_{\Lambda, \mu, \beta, i}^{\vec{m}} X_{m_{1}} \ldots X_{m_{n}}=\frac{1}{n !} \sum_{\sigma \in S_{n}} B_{j \beta} D_{i j}^{\Lambda}(\sigma) \sigma \mathbf{X}^{\mu} \sigma^{-1}
$$

We then find the orthogonality we expect, up to a normalisation factor

$$
\sum_{\vec{m}} C_{\Lambda, \mu, \beta, i}^{\vec{m}} C_{\Lambda^{\prime}, \mu^{\prime}, \beta^{\prime}, i^{\prime}}^{\vec{m}}=\delta_{\Lambda \Lambda^{\prime}} \delta_{\mu \mu^{\prime}} \delta_{\beta \beta^{\prime}} \delta_{i i^{\prime}} \frac{\left|H_{\mu}\right|}{n ! d_{\Lambda}}
$$

\subsection{The higher spin group}

The free theory of $\mathcal{N}=4 \mathrm{SYM}$ is invariant under an infinite dimensional group $H S(2,2 \mid 4)$ known as the higher spin group. In the interacting theory this is broken to the superconformal group $S U(2,2 \mid 4)$ but it can nevertheless be useful for some applications (eg possible relations via AdS/CFT to a possible 'tensionless limit' of string theory) to consider this enlarged group. When restricted to the $S L(2)$ sector the higher spin group is known as $H S(1,1)$. Operators form lowest weight representations of $H S(1,1)$ (which further decompose into an infinite number of lowest weight representations of $S L(2)$.) The lowest weight states of these representations were decscribed in [27]. In terms of the oscillators introduced in section 2.1, the higher spin algebra is spanned by the generators

$$
J_{p, q}=\sum_{i}\left(a_{i}^{\dagger}\right)^{p}\left(a_{i}\right)^{q}
$$


which clearly contains the $S L(2)$ algebra (16) $)$.

If we consider truncating the fundamental fields so that we only consider states $|m\rangle=$ $\left(a^{\dagger}\right)^{m}|0\rangle$ for $m \leq M-1$ then the higher spin group truncates to $U(M)$. Therefore the covariant canonical form corresponding to the higher spin group is simply the $M \rightarrow \infty$ limit of that in the previous subsection.

Therefore the results of the previous subsection generalise naturally to the higher spin case. Irreducible representations of the higher spin group are specified by Young Tableaux, $\Lambda_{1}$, (as observed in [27]). We have

$$
\begin{aligned}
V_{F}^{\otimes n} & =\bigoplus_{\Lambda_{1} \vdash n} V_{\Lambda_{1}}^{H S} \otimes V_{\Lambda_{1}}^{S_{n}} \\
& =\bigoplus_{\Lambda_{1}, \Lambda} V_{\Lambda}^{S L(2)} \otimes V_{\Lambda, \Lambda_{1}}^{\mathrm{Com}\left(S L(2) \times S_{n}\right)} \otimes V_{\Lambda_{1}}^{\left(S_{n}\right)}
\end{aligned}
$$

The first line is the standard Schur-Weyl duality for $U(M)$ in the limit $M \rightarrow \infty$. Each higher spin representation, $\Lambda_{1}$, then decomposes further into an $S L(2)$ irrep $\Lambda$ and the commutant. The Clebsch gving the first line is given by (88). The canonical covariant operators which form irreps of the higher spin group are given by (89) with $X_{m}$ replaced by $(1 / m !) \partial^{m} X$.

\subsection{The $S O(6)$ sector}

We have 6 hermitian scalar matrices in $\mathcal{N}=4$ SYM, transforming in the fundamental of $S O(6)$. We know from the general discussion in Section 4 that the $S O(6)$ covariant diagonalisation of free field correlators will be solved once we have solved the Clebsch-Gordan problem for $S O(6) \times S_{n}$ in $V^{\otimes n}$. Here $V$ is the fundamental of $S O(6)$.

$$
\begin{aligned}
V^{\otimes n} & =\bigoplus_{\Lambda_{1}} V_{\Lambda_{1}}^{\left(S_{n}\right)} \otimes V_{\Lambda_{1}}^{G L(6)} \\
& =\bigoplus_{\Lambda_{1}, \Lambda_{2}} V_{\pi\left(\Lambda_{2}\right)}^{(S O(6))} \otimes V_{\Lambda_{1}, \Lambda_{2}} \otimes V_{\Lambda_{1}}^{\left(S_{n}\right)}
\end{aligned}
$$

We first decompose the $n$-fold tensor space according to the $S_{n}$ symmetry. The SchurWeyl dual of $S_{n}$ is $G L(6)$ hence the decomposition in the first line. In the second line, we decompose the $G L(6)$ representations to $S O(6)$ representations. The dimension of the multiplicity space $V_{\Lambda_{1}, \Lambda_{2}}$ is given by

$$
\operatorname{Dim} V_{\Lambda_{1}, \Lambda_{2}}=\sum_{\delta} g\left(\Lambda_{2}, 2 \delta ; \Lambda_{1}\right)
$$

$\Lambda_{1}$ is a Young diagram with $n$ boxes, $2 \delta$ is a partition with even parts, i.e. Young diagram with even row lengths. The sum above includes a sum over $k \geq 0$, where $2 k$ is the number of boxes in $2 \delta$ and $n-2 k$ is the number of boxes in $\Lambda_{2}$.

The representations of $G L(6)$ are labelled by Young diagrams with row lengths $\lambda_{1} \geq \lambda_{2} \geq$ $\cdots \lambda_{6} \geq 0$. The representations of $S O(6)$ are labelled by $\lambda_{1} \geq \lambda_{2} \geq\left|\lambda_{3}\right| \geq 0$. The last label $\lambda_{3}$ 
can be positive or negative. For $\lambda_{3}=0$ the irreps are constructed by symmetrising according to the Young diagram and projecting out traces. When $\left|\lambda_{3}\right|>0$ the corresponding operation of Young-symmetrising and removing traces leaves us with a reducible representation, which is a direct sum of irreps. $\left(\lambda_{1}, \lambda_{2}, \lambda_{3}\right) \oplus\left(\lambda_{1}, \lambda_{2},-\lambda_{3}\right)$. The operation $\pi$ which appears in (94), when it acts on any $G L(6)$ Young diagram $\Lambda_{1}$ gives either zero or a Young diagram obeying the $S O(6)$ constraints. It is defined in terms of an operation on Young diagrams in [31].

We have arrived above at the $S O(6) \times S_{n}$ decomposition by first decomposing into $G L(6) \times$ $S_{n}$, then reducing the $G L(6)$ to $S O(6)$. We can equally start by decomposing in terms of $S O(6) \times E_{6}(n)$ where $E_{6}(n)$ is the commutant of $S O(6)$ in $V^{\otimes n}$ described for example in [32. A subsequent decomposition of $E_{6}(n)$ to $S_{n}$ should yield the same result as (94). This follows from general theorems on double commutants which assert that if $A$ is a subalgebra of $B$, and $\operatorname{End}(B) \subset \operatorname{End}(A)$ are their commutants in some vector space, then the reduction multiplicities for irreps of $B \rightarrow A$ coincide with those of $\operatorname{End}(A) \rightarrow \operatorname{End}(B)$ (see [17] ). In this case the reduction multiplicities of $G L(6) \rightarrow S O(6)$ coincide with those of $E_{6}(n) \rightarrow S_{n}$.

\subsection{The $S O(4,2)$ sector}

In considering the sector of a scalar field $X$ with all four derivatives acting on it, we can use the $S O(4,2)$ symmetry. Generalizing the linear combinations $A_{a}^{\dagger}$ of oscillators which generate the lowest weights in the $S L(2)$ sector, we now have $A_{a \lambda}^{\dagger}$ where $\lambda$ is an index in the fundamental of $S O(4) \subset S O(4,2)$ and as before $a$ is in the hook representation $V_{H}=[n-1,1]$ of $S_{n}$. Lowest weights annihilated by $K_{\lambda}$, with $k$ derivatives acting on $n$-field composites can be constructed from oscillators of the form

$$
A_{a_{1} \lambda_{1}}^{\dagger} A_{a_{2} \lambda_{2}}^{\dagger} \cdots A_{a_{k} \lambda_{k}}^{\dagger}|0\rangle
$$

The simplest class of such LWS are those in which the indices $\left(\lambda_{1}, \lambda_{2}, \cdots, \lambda_{k}\right)$ are taken to be a symmetric traceless $S O(4)$ tensor corresponding to the $S O(4)$ Young diagram $[k]$. These states satisfy a type of extremality condition $L_{0}=n+k$. More generally we will have states of the form (96) which involve contractions of the $\lambda_{i}$. In these cases we have to mod out by the equations of motion, which leads to a projection of the $\operatorname{Sym}\left(V_{H} \otimes V_{H}\right)$

representation of $A_{a_{1} \lambda}^{\dagger} A_{a_{2} \lambda}^{\dagger}$ to the $S_{n}$ representation $[n-2,2]$. This has dimension $\frac{n(n-3)}{2}$ which is the number obtained by subtraction of $n$, for the equations of motion, from the dimension $\frac{n(n-1)}{2}$ of $\operatorname{Sym}\left(V_{H} \otimes V_{H}\right)$. Work on a complete solution of the diagonalisation in this sector, using the above facts to give a symmetric group description of the $S O(4,2) \times S_{n}$ Clebsch-Gordans, is in progress. It is clear that the symmetric $S O(4)$ operators involving the contractions will have $L_{0}>n+k$. The 'extremal' operators mentioned above will be useful in the comparison to excitations of half-BPS giants in Section 7 . 


\subsection{Fields carrying reps of product groups}

Suppose the global symmetry group has the form $G_{1} \times G_{2}$. We consider a field $\Psi_{k, m}$ where $k$ is an index transforming under irrep $V_{1}$ of $G_{1}$ and $m$ transforms under irrep $V_{2}$ of $G_{2}$. Consider the covariant operator

$$
\left(\mathcal{O}_{k_{1}, m_{1} ; k_{2}, m_{2} ; \cdots ; k_{n}, m_{n}}\right)_{J}^{I} \equiv\left(\Psi_{k_{1}, m_{1}}\right)_{j_{1}}^{i_{1}}\left(\Psi_{k_{2}, m_{2}}\right)_{j_{2}}^{i_{2}} \cdots\left(\Psi_{k_{n}, m_{n}}\right)_{j_{n}}^{i_{n}}
$$

Fields with $n$ factors transform under the irrep $\left(V_{1} \otimes V_{2}\right)^{\otimes n}$. With $\sigma \in S_{n}$ acting simultaneously on $V_{1}$ and $V_{2}$, the commutant of $G_{1} \times G_{2}$ contains $S_{n}$. We can consider the group $G_{1} \times G_{2} \times S_{n}$ acting on the $n$-field composites. Correspondingly there is a decomposition of the $n$-fold tensor product into irreps. of $G_{1} \times G_{2} \times S_{n}$. The irreps are related to the product states as

$$
\left|\Lambda_{1}, M_{\Lambda_{1}}, \Lambda_{2}, M_{\Lambda_{2}}, \Lambda_{3}, m_{\Lambda_{3}}, \tau\right\rangle=C_{\Lambda_{1}, M_{\Lambda_{1}, \Lambda_{2}, M_{\Lambda_{2}}, \Lambda_{3}, m_{\Lambda_{3}}, \tau}^{\vec{k}, \vec{k}}|\vec{m}\rangle} \mid \vec{k}
$$

$\Lambda_{1}$ is an irrep of $G_{1}, \Lambda_{2}$ of $G_{2}$ and $\Lambda_{3}$ of $S_{n}$. Conversely

$$
|\vec{k}, \vec{m}\rangle=C_{\vec{k}, \vec{m}}^{\Lambda_{1}, M_{\Lambda_{1}}, \Lambda_{2}, M_{\Lambda_{2}}, \Lambda_{3}, m_{\Lambda_{3}}, \tau}\left|\Lambda_{1}, M_{\Lambda_{1}}, \Lambda_{2}, M_{\Lambda_{2}}, \Lambda_{3}, m_{\Lambda_{3}}, \tau\right\rangle
$$

In terms of vector spaces this decomposition is

$$
\left(V_{1} \otimes V_{2}\right)^{\otimes n}=\bigoplus_{\Lambda_{1}, \Lambda_{2}, \Lambda_{3}} V_{\Lambda_{1}}^{G_{1}} \otimes V_{\Lambda_{2}}^{G_{2}} \otimes V_{\Lambda_{3}}^{S_{n}} \otimes V_{\Lambda_{1}, \Lambda_{2}, \Lambda_{3}}^{\mathrm{Com}\left(G_{1} \times G_{2} \times S_{n}\right)}
$$

$\tau$ over the multiplicity space $V_{\Lambda_{1}, \Lambda_{2}, \Lambda_{3}}^{\operatorname{Com}\left(G_{1} \times G_{2} \times S_{n}\right)}$.

The orthogonality of Clebsch-Gordan coefficients is the same as before and if we define operators

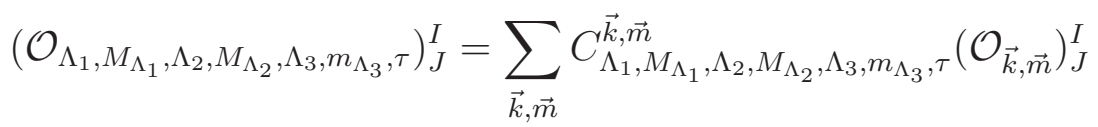

then the covariant two-point function is diagonal in the $\left(\Lambda_{1}, M_{\Lambda_{1}}, \Lambda_{2}, M_{\Lambda_{2}}, \Lambda_{3}, \tau\right)$ indices, exactly analogous to the single group case in equation (77).

\subsubsection{Product Clebsch in terms of single group Clebschs}

Another way that we could organise $\left(V_{1} \otimes V_{2}\right)^{\otimes n}$, in contrast to the $G_{1} \times G_{2} \times S_{n}$ decomposition in (100), is in terms of the separate groups

$$
\begin{aligned}
\left(V_{1} \otimes V_{2}\right)^{\otimes n} & =V_{1}^{\otimes n} \otimes V_{2}^{\otimes n} \\
& =\left(\bigoplus_{\Lambda_{1}, \Lambda_{4}} V_{\Lambda_{1}}^{G_{1}} \otimes V_{\Lambda_{4}}^{S_{n}} \otimes V_{\Lambda_{1}, \Lambda_{4}}^{\mathrm{Com}\left(G_{1} \times S_{n}\right)}\right) \otimes\left(\bigoplus_{\Lambda_{2}, \Lambda_{5}} V_{\Lambda_{2}}^{G_{2}} \otimes V_{\Lambda_{5}}^{S_{n}} \otimes V_{\Lambda_{2}, \Lambda_{5}}^{\mathrm{Com}\left(G_{2} \times S_{n}\right)}\right)
\end{aligned}
$$

We use the Clebsch $C_{\vec{k}}^{\Lambda_{1}, M_{\Lambda_{1}}, \Lambda_{4}, m_{\Lambda_{4}}, \tau_{1}}$ for $G_{1}$ and $C_{\vec{m}}^{\Lambda_{2}, M_{\Lambda_{2}}, \Lambda_{5}, m_{\Lambda_{5}}, \tau_{2}}$ for $G_{2}$. Given the simultaneous action of $S_{n}$ on $\left(V_{1} \otimes V_{2}\right)^{\otimes n}$, to connect this decomposition with that in (100) we 
tensor together the two $S_{n}$ irreps $V_{\Lambda_{4}}^{S_{n}}$ and $V_{\Lambda_{5}}^{S_{n}}$ to get the irrep of the simultaneous $S_{n}$ action $V_{\Lambda_{3}}^{S_{n}}$

$$
V_{\Lambda_{4}}^{S_{n}} \otimes V_{\Lambda_{5}}^{S_{n}}=\bigoplus_{\Lambda_{3}} V_{\Lambda_{3}}^{S_{n}} C\left(\Lambda_{4}, \Lambda_{5} ; \Lambda_{3}\right)
$$

$C\left(\Lambda_{4}, \Lambda_{5} ; \Lambda_{3}\right)$ counts the number of times $V_{\Lambda_{3}}^{S_{n}}$ appears in the $S_{n}$ tensor product $V_{\Lambda_{4}}^{S_{n}} \otimes V_{\Lambda_{5}}^{S_{n}}$. This construction shows us how to write down the relation between the $G_{1} \times G_{2} \times S_{n}$ Clebsch and the $\left(G_{1} \times S_{n}\right) \times\left(G_{2} \times S_{n}\right)$ Clebschs

$$
C_{\vec{k}, \vec{m}}^{\Lambda_{1}, M_{\Lambda_{1}}, \Lambda_{2}, M_{\Lambda_{2}}, \Lambda_{3}, m_{\Lambda_{3}}, \tau}=C_{\vec{k}}^{\Lambda_{1}, M_{\Lambda_{1}}, \Lambda_{4}, m_{\Lambda_{4}}, \tau_{1}} C_{\vec{m}}^{\Lambda_{2}, M_{\Lambda_{2}}, \Lambda_{5}, m_{\Lambda_{5}}, \tau_{2}} C^{\tau_{3} \Lambda_{3}}{ }_{m_{\Lambda_{3}}}^{\Lambda_{m_{4}}} m_{\Lambda_{4}}{ }_{m_{\Lambda_{5}}}^{\Lambda_{5}}
$$

The $S_{n}$ Clebsch-Gordan coefficient $\begin{array}{cccc}C_{3} \Lambda_{3} & \Lambda_{4} & \Lambda_{5} \\ m_{\Lambda_{3}} & m_{\Lambda_{4}} & m_{\Lambda_{5}}\end{array}$ gives the change of basis for the decomposition in (102); it maps the states of the reps in $V_{\Lambda_{4}}^{S_{n}} \otimes V_{\Lambda_{5}}^{S_{n}}$ to those in $V_{\Lambda_{3}}^{S_{n}}$. $\tau_{3}$ labels the $C\left(\Lambda_{4}, \Lambda_{5} ; \Lambda_{3}\right)$ degeneracy. The $\tau$ which labels the product group commutant $V_{\Lambda_{1}, \Lambda_{2}, \Lambda_{3}}^{\mathrm{Com}\left(G_{1} \times G_{2} \times S_{n}\right)}$ is now a combination of the separate group multiplicities and the $S_{n}$ tensor label $\tau_{3}: \tau=\left(\tau_{1}, \tau_{2}, \tau_{3}\right)$.

$$
V_{\Lambda_{1}, \Lambda_{2}, \Lambda_{3}}^{\operatorname{Com}\left(G_{1} \times G_{2} \times S_{n}\right)}=\bigoplus_{\Lambda_{4}, \Lambda_{5}} V_{\Lambda_{1}, \Lambda_{4}}^{\operatorname{Com}\left(G_{1} \times S_{n}\right)} \otimes V_{\Lambda_{2}, \Lambda_{5}}^{\operatorname{Com}\left(G_{2} \times S_{n}\right)} C\left(\Lambda_{4}, \Lambda_{5} ; \Lambda_{3}\right)
$$

In the special case when the gauge group is $U(1)$, so that the fields commute, $\Lambda_{3}\left(S_{n}\right)$ is the trivial representation. This forces $\Lambda_{4}\left(S_{n}\right)=\Lambda_{5}\left(S_{n}\right)$. The same thing applies when considering bosonic oscillators carrying indices of $G_{1} \times G_{2}$.

\section{Gauge invariant operators}

We have organised $n$ copies of the fundamental fields in terms of representations of the global symmetry group $G$.

$$
\mathcal{O}^{\Lambda, M_{\Lambda}, \Lambda_{1}, m_{\Lambda_{1}}, \tau}=C_{\Lambda, M_{\Lambda}, \Lambda_{1}, m_{\Lambda_{1}}, \tau}^{\vec{m}} W_{m_{1}} \otimes W_{m_{2}} \otimes \cdots \otimes W_{m_{n}}
$$

We now introduce the $U(N)$ gauge group indices

$$
\left(W_{m}\right)_{j}^{i}
$$

and view the $W_{m}$ as operators on the fundamental representation $V_{N}$ of $U(N)$. To form gauge-invariant operators we multiply these matrices together and then take products of traces organised by the symmetric group element $\alpha \in S_{n}$

$$
\operatorname{tr}\left(\alpha W_{m_{1}} \otimes W_{m_{2}} \otimes \cdots \otimes W_{m_{n}}\right)=\left(W_{m_{1}}\right)_{i_{\alpha(1)}}^{i_{1}}\left(W_{m_{2}}\right)_{i_{\alpha(2)}}^{i_{2}} \cdots\left(W_{m_{n}}\right)_{i_{\alpha(n)}}^{i_{n}}
$$

where the trace is being taken in $V_{N}^{\otimes n}$. We can reorganise these in terms of representations $R$ of $U(N)$ using the Schur-Weyl dual $S_{n}$ representation matrices $D_{i j}^{R}(\alpha)$

$$
\frac{1}{n !} \sum_{\alpha \in S_{n}} D_{i j}^{R}(\alpha) \operatorname{tr}\left(\alpha W_{m_{1}} \otimes W_{m_{2}} \cdots \otimes W_{m_{n}}\right)
$$


As a representation of $U(N), R$ has at most $N$ rows.

Combining the free $S_{n}$ indices with an $S_{n}$ Clebsch-Gordan coefficient gives a gauge invariant operator

$$
\begin{aligned}
\mathcal{O}^{\Lambda, M_{\Lambda}, \Lambda_{1}, \tau, R, \tau_{\Lambda_{1}, R}} & =C^{\tau_{\Lambda_{1}, R}}{\underset{m}{m_{\Lambda_{1}}}{ }_{i} \underset{j}{R}{ }_{j}}_{C_{\Lambda, M_{\Lambda}, \Lambda_{1}, m_{\Lambda_{1}}, \tau}} \frac{1}{n !} \sum_{\alpha \in S_{n}} D_{i j}^{R}(\alpha) \operatorname{tr}\left(\alpha W_{m_{1}} \otimes \cdots \otimes W_{m_{n}}\right) \\
& =C^{\tau_{\Lambda_{1}, R}}{\underset{m}{M_{\Lambda_{1}}}{ }_{i}{ }_{i}}_{j}^{R} \frac{1}{n !} \sum_{\alpha \in S_{n}} D_{i j}^{R}(\alpha) \operatorname{tr}\left(\alpha \mathcal{O}^{\Lambda, M_{\Lambda}, \Lambda_{1}, m_{\Lambda_{1}}, \tau}\right)
\end{aligned}
$$

We can invert the Clebschs to recover from these operators the basic gauge invariant operators in (107). This means that our new basis is complete. It also counts correctly at finite $N$, as demonstrated in the next section. Furthermore, following the methods of [15], it is fully diagonal in all its labels.

$$
\begin{aligned}
& \left\langle\mathcal{O}^{\Lambda, M_{\Lambda}, \Lambda_{1}, \tau, R, \tau_{\Lambda_{1}, R}}\left(\mathcal{O}^{\dagger}\right)^{\Lambda^{\prime}, M_{\Lambda^{\prime}}^{\prime}, \Lambda_{1}^{\prime}, \tau^{\prime}, R^{\prime}, \tau_{\Lambda_{1}^{\prime}, R^{\prime}}^{\prime}}\right\rangle
\end{aligned}
$$

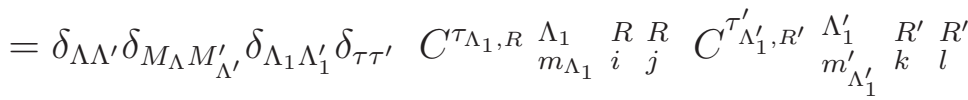

$$
\begin{aligned}
& \frac{1}{n !} \sum_{\alpha \in S_{n}} D_{i j}^{R}(\alpha) \frac{1}{n !} \sum_{\alpha^{\prime} \in S_{n}} D_{k l}^{R^{\prime}}\left(\alpha^{\prime}\right) \sum_{\sigma \in S_{n}} D_{m_{\Lambda_{1}} m_{\Lambda_{1}^{\prime}}^{\prime}}^{\Lambda_{1}}(\sigma) \operatorname{tr}\left(\alpha \sigma \alpha^{\prime} \sigma^{-1}\right) \\
& =\delta_{\Lambda \Lambda^{\prime}} \delta_{M_{\Lambda} M_{\Lambda^{\prime}}^{\prime}} \delta_{\Lambda_{1} \Lambda_{1}^{\prime}} \delta_{\tau \tau^{\prime}} \delta_{R R^{\prime}} \delta_{\tau_{\Lambda_{1}, R} \tau_{\Lambda_{1}^{\prime}, R^{\prime}}^{\prime}} \frac{n ! d_{\Lambda_{1}}}{d_{R}^{2}} \operatorname{Dim} R
\end{aligned}
$$

In the second line we have used the canonical covariant correlator (77). $\operatorname{Dim} R$ is the $U(N)$ dimension of $R$.

\subsection{Finite $N$ counting}

We show here that the operators defined in equation (109) count correctly for finite $N$. The finite $N$ partition function is given in terms of the single letter partition function $f(\mathbf{x})$, for bosonic $\mathbf{x}$, by an integral over the $U(N)$ matrix $U$ [33, 34].

$$
\mathcal{Z}=\int[d U] \exp \left\{\sum \frac{1}{m} f\left(\mathbf{x}^{m}\right) \operatorname{tr}\left(U^{\dagger}\right)^{m} \operatorname{tr} U^{m}\right\}
$$

$f(\mathbf{x})$ is the character of the fundamental representation $V_{F}$. For $U(3)$ it is

$$
f\left(\mathbf{x}^{m}\right)=x_{1}^{m}+x_{2}^{m}+x_{3}^{m}
$$

and for $S L(2)$ it is

$$
f\left(\mathbf{x}^{m}\right)=\frac{q^{m}}{1-q^{m}}
$$

Now we perform the group integration for $U(N)$ following [21] (see also [35]). If we expand out

$$
\exp \left\{\sum \frac{1}{m} f\left(\mathbf{x}^{m}\right) \operatorname{tr}\left(U^{\dagger}\right)^{m} \operatorname{tr} U^{m}\right\}
$$


we get

$$
\sum_{n} \sum_{C_{\mathbf{i}} \in S_{n}} \prod_{j=1}^{n}\left(f\left(\mathbf{x}^{j}\right)\right)^{i_{j}} \frac{1}{j^{i_{j}} i_{j} !} \operatorname{tr}\left(C_{\mathbf{i}} U\right) \operatorname{tr}\left(C_{\mathbf{i}} U^{\dagger}\right)
$$

where $C_{\mathbf{i}}$ is a partition of $n$ or a conjugacy class of $S_{n}$ with $i_{1}$ 1-cycles, $i_{2} 2$-cycles, $\ldots i_{n}$ $n$-cycles. In $\frac{1}{j^{i} i_{j} \text { ! }}$ the $j^{i_{j}}$ comes from the $\frac{1}{m}$ in (114) and the $i_{j}$ ! comes from $\exp (x)=\sum_{k} \frac{1}{k !} x^{k}$.

Using the identity $\operatorname{tr}\left(C_{\mathbf{i}} U\right)=\sum_{R(U(N))} \chi_{R}\left(C_{\mathbf{i}}\right) \chi_{R}(U)$ and the group integral

$$
\int[d U] \chi_{R}(U) \chi_{R^{\prime}}\left(U^{\dagger}\right)=\delta_{R R^{\prime}}
$$

we get the finite $N$ partition function

$$
\mathcal{Z}=\sum_{n} \sum_{R(U(N))} \sum_{C_{\mathbf{i}} \in S_{n}} \prod_{j=1}^{n}\left(f\left(\mathbf{x}^{j}\right)\right)^{i_{j}} \frac{1}{j^{i_{j}} i_{j} !} \chi_{R}\left(C_{\mathbf{i}}\right) \chi_{R}\left(C_{\mathbf{i}}\right)
$$

Now if we treat $\mathbf{x}$ as a diagonal matrix (for $U(3)$ we have $\left(x_{1}, x_{2}, x_{3}\right)$ on the diagonal, for $S L(2)$ we have $\left.\left(q, q^{2}, q^{3}, \ldots\right)\right)$ and use

$$
\prod_{j=1}^{n}\left(f\left(\mathbf{x}^{j}\right)\right)^{i_{j}}=\operatorname{tr}\left(C_{\mathbf{i}} \mathbf{x}\right)=\sum_{\Lambda_{1}\left(S_{n}\right)} \chi_{\Lambda_{1}}\left(C_{\mathbf{i}}\right) \chi_{\Lambda_{1}}(\mathbf{x})
$$

then we get

$$
\mathcal{Z}=\sum_{n} \sum_{R(U(N))} \sum_{\Lambda_{1}\left(S_{n}\right)} \chi_{\Lambda_{1}}(\mathbf{x}) C\left(R, R, \Lambda_{1}\right)
$$

where $C\left(R, R, \Lambda_{1}\right)$ is the number of possible $\tau_{\Lambda_{1}, R}$ multiplicities in (109), i.e. the number of times $\Lambda_{1}$ appears in the symmetric group tensor product $R \otimes R 2$ As representations of $U(N)$, we only sum over Young diagrams $R$ with at most $N$ rows.

We have treated the global symmetry group here as $G L(\infty)$. A further decomposition into irreps. of $G$ gives

$$
V_{\Lambda_{1}}^{G L(\infty)}=\sum_{\Lambda} V_{\Lambda}^{G} \otimes V_{\Lambda, \Lambda_{1}}
$$

When we do this we finally see that the operators in (109) provide this counting.

$$
\mathcal{Z}=\sum_{n} \sum_{R(U(N))} \sum_{\Lambda(G)} \sum_{\Lambda_{1}\left(S_{n}\right)} d_{\Lambda, \Lambda_{1}} \chi_{\Lambda}(\mathbf{x}) C\left(R, R, \Lambda_{1}\right)
$$

where $\chi_{\Lambda}(\mathbf{x})$ is now a $G$ character and $d_{\Lambda, \Lambda_{1}}$ is the dimension of $V_{\Lambda, \Lambda_{1}}$ labelled by the $\tau$ index in (109).

\footnotetext{
${ }^{2} C(R, S, T)=\frac{1}{n !} \sum_{\sigma \in S_{n}} \chi_{R}(\sigma) \chi_{S}(\sigma) \chi_{T}(\sigma)$ and $\prod_{j=1}^{n} \frac{1}{j^{i j} i_{j} !}=\frac{\left|C_{\mathbf{i}}\right|}{n !}$ where $\left|C_{\mathbf{i}}\right|$ is the size of the class $C_{\mathbf{i}}$.
} 


\section{Worldvolume excitations of giants and gauge invari- ant operators}

\subsection{Worldvolume excitations: review and comments}

We review and comment on some results from [22] on the worldvolume excitations of halfBPS giant gravitons. Consider 3-brane giants expanding in the $A d S^{5}$. Use coordinates $\left(t, v_{1}, v_{2}, v_{3}, v_{4}\right)$ for the AdS where we have a metric

$$
d s^{2}=-\left(1+\sum_{k=1}^{4} v_{k}^{2}\right) d t^{2}+L^{2}\left(\delta_{i j}+\frac{v_{i} v_{j}}{\left(1+\sum_{k} v_{k}^{2}\right)}\right) d v_{i} d v_{j}
$$

$L$ is the $A d S_{5}$ or $S^{5}$-radius. The $S^{5}$ can be described in analogous coordinates

$$
d s^{2}=L^{2}\left[\left(1-\sum_{k=1}^{4} y_{k}^{2}\right) d \phi^{2}+\left(\delta_{i j}+\frac{y_{i} y_{j}}{1-\sum_{k} y_{k}^{2}}\right) d y_{i} d y_{j}\right]
$$

In global coordinates the AdS metric is

$$
d s^{2}=-\left(1+\frac{r^{2}}{L^{2}}\right) d t^{2}+\frac{d r^{2}}{\left(1+\frac{r^{2}}{L^{2}}\right)}+r^{2} d \Omega_{3}^{2}
$$

It is also useful to write the $S^{5}$ metric as

$$
d s^{2}=L^{2}\left(d \theta^{2}+\cos ^{2} \theta d \phi^{2}+\sin ^{2} \theta d \Omega_{3}^{2}\right)
$$

The AdS-giant graviton solution has

$$
\begin{aligned}
& \phi=\omega_{0} t \\
& \omega_{0}=\frac{1}{L} \\
& P_{\phi}=N\left(\frac{r_{0}}{L}\right)^{2}
\end{aligned}
$$

and the half-BPS property guarantees the energy is $E=\frac{P_{\phi}}{L}$. The brane worldvolume coordinates are $\tau, \sigma_{1}, \sigma_{2}, \sigma_{3}$. The coordinate $\tau$ is identified with the global time $t$. The $\sigma_{1}, \sigma_{2}, \sigma_{3}$ are identified with angles in $A d S$.

The fluctuations are expanded as

$$
\begin{aligned}
& r=r_{0}+\epsilon \delta r\left(\tau, \sigma_{1}, \sigma_{2}, \sigma_{3}\right) \\
& \phi=\omega_{0} \tau+\epsilon \delta \phi\left(\tau, \sigma_{1}, \sigma_{2}, \sigma_{3}\right) \\
& y_{k}=\epsilon \delta y_{k}\left(\tau, \sigma_{1}, \sigma_{2}, \sigma_{3}\right)
\end{aligned}
$$

These perturbations are expanded in spherical harmonics.

$$
\delta r\left(\tau, \sigma_{i}\right)=\tilde{\delta} r e^{-i \omega \tau} Y_{l}\left(\tau, \sigma_{i}\right)
$$




$$
\begin{aligned}
& \delta \phi\left(\tau, \sigma_{i}\right)=\tilde{\delta} \phi e^{-i \omega \tau} Y_{l}\left(\tau, \sigma_{i}\right) \\
& \delta y_{k}=\tilde{\delta} y_{k} e^{-i \omega \tau} Y_{l}\left(\tau, \sigma_{i}\right)
\end{aligned}
$$

The $\left(\phi, y_{k}\right)$ are coordinates for the sphere $S^{5}$. The $Y_{l}$ are spherical harmonics on $S^{3} \subset A d S_{5}$. They are symmetric traceless representations of $S O(4)$. They have a quadratic Casimir $l(l+2)$ for the symmetric traceless representation of dimension $(l+1)^{2}$. The frequencies of these oscillations are calculated from the linearized equations of motion of the brane actions

$$
S=S_{D B I}+S_{C S}
$$

They lead (after a small simplification of expressions in [22]) to three solutions

$$
\begin{aligned}
& \omega_{-}=\frac{l}{L} \\
& \omega_{+}=\frac{l+2}{L} \\
& \omega=\frac{l+1}{L}
\end{aligned}
$$

The modes with frequencies $\omega_{ \pm}$are related to linear combinations $\tilde{\delta} r, \tilde{\delta} \phi$. The frequency $\omega$ is related to four modes $\tilde{\delta} v_{m}$ which transform in the fundamental of $S O(4)$ in $S O(6)$. It is very interesting that these are all integer multiples of the AdS-scale and approach $\omega=l / L$ in the large $l$ limit. Note also that $\omega$ is the frequency for oscillations in $t$, the global time of AdS. The energies of the fluctuating giant gravitons are given by $E=\frac{n}{L}+\omega$ where $n$ is the angular momentum of the background giant. The energy is related to scaling dimension in the dual CFT [3]. These energy spacings in integer units of $\frac{1}{L}$ are precisely the sort of spacings we get in free Yang Mills theory. Taking large angular momentum limits as a way to reach a classical regime where strong and weak coupling coupling can be compared directly is familiar from [38].

The $Y_{l, m}$ are representations of $S O(4)$. Specifying the eigenvalues of the Cartan amounts to fixing two spins $S_{1}, S_{2}$. The $S L(2)$ sector of gauge theory operators we considered, involving multitraces of $\partial_{1+i 2}^{S} X^{n}$ corresponds to rotations in a fixed plane. This means that in each space of spherical harmonics of given $l$ we are looking at a single state. Now if we consider a second quantization in the field theory of the branes, we would introduce a Fock space generator $\alpha_{l}^{\dagger}$ for each spherical harmonic. This has energy $l / L$ above the background energy of the brane. General states look like

$$
\alpha_{1}^{\dagger k_{1}} \alpha_{2}^{\dagger k_{2}} \cdots|0\rangle
$$

The number of states at excitation energy $k$ is the number of ways of writing $k=k_{1}+2 k_{2}+$ $\cdots=\sum_{i} k_{i} l_{i}$ which is the number of partitions of $k$. When we restore the full $S O(4)$ we have states of the form

$$
\alpha_{l_{1}, m_{1}}^{\dagger} \alpha_{l_{2}, m_{2}}^{\dagger} \cdots|0\rangle
$$


In this case it is useful to restrict attention to the symmetric traceless representations $[k]$ of $S O(4)$ with excitation energy equal to $k$. In this case, the number of excited states of total energy $L_{0}=n+k$ is again given by partitions of $k$. In the discussion below we will show that that there is an easy way to get these states from the gauge theory. In greater generality we should consider states of the form

$$
\alpha_{l_{1}, m_{1}, I_{1}}^{\dagger} \alpha_{l_{2}, m_{2}, I_{2}}^{\dagger} \cdots|0\rangle
$$

where I's are indices running from 1 to 6 which label the six eigenmodes built from (128). Four of these are in the fundamental of $S O(4) \subset S O(6)$. The fact that the excitation energies are spaced in units of $\frac{1}{L}$ (rather than in units of the brane size) was a bit of a surprise, discussed at length in [22]. An important point is that the kind of integer spacing in (130) is exactly what we have in free Yang Mills limit of the dual CFT. We will see below that this Fock space structure of orthogonal states emerges indeed from the construction of gauge invariant operators in the free dual Yang Mills theory. A connection between excited giant gravitons and the formulae for excitation energies (130) was made in [36]. The unravelling of the Fock space structure of giant graviton worldvolume field theory from gauge invariant operator counting given below is new.

\subsection{Comparison to gauge invariant operators}

We have constructed, in section 2, the lowest weights of the $S L(2)$ sector by mapping states

$$
A_{a_{1}}^{\dagger} A_{a_{2}}^{\dagger} \cdots A_{a_{l}}^{\dagger}|0\rangle
$$

in an oscillator construction of $S L(2)$ to gauge theory operators. The index a transforms in the hook representation $[n-1,1]$ of $S_{n}$. The $A^{\dagger}$ 's are bosons so we are looking at the symmetric tensor product of the hook. These were constructed as lowest weight states generated by $P_{1 i}$ which forms part of the $S O(4,2)$ conformal algebra. These excitations correspond to exciting one spin inside AdS (for more details on the geometry of multiple spins see for example [37] in the context of spinning strings), hence to states of the form (131). After describing how to lift this to more general $S O(4,2)$ states, we will show that the counting in the case of single giants agrees with the bulk analysis reviewed above. Note for now that the above states transform in $\operatorname{Sym}\left(V_{H}^{\otimes k}\right)$ of $S_{n}$.

When we consider the full $S O(4,2)$ symmetry, we have additional generators $K_{\lambda}$ forming the fundamental of $S O(4)$. Correspondingly we have $P_{\lambda}$ transforming in the fundamental of $S O(4)$. When we consider lowest weight states annihilated by all the $K_{\lambda}$, we have states of the form

$$
A_{a_{1} \lambda_{1}}^{\dagger} A_{a_{2} \lambda_{2}}^{\dagger} \cdots A_{a_{k} \lambda_{k}}^{\dagger}|0\rangle
$$

Among these LWS are those transforming in the symmetric traceless representation of $S O(4)$ associated with the symmetric Young diagram $[k]$ and with energy $L_{0}=n+k$. As discussed 
in 5.5, these are a simple class of states which do not require projecting out of states due to the equations of motion, which require setting $P_{\lambda} P_{\lambda}$ to zero. Since the $\lambda^{\prime}$ 's are symmetrised, and the $A^{\dagger}$ are bosons, the indices $a_{1}, a_{2}, \cdots, a_{l}$ are symmetric, i.e. we have the symmetric $k$-fold tensor power of the hook representation $[n-1,1]$ of $S_{n}$. Orthonormal states in this sector are then written as

$$
C_{\lambda_{1} \cdots \lambda_{k}}^{[k], M_{[k]}} C_{a_{1} \ldots a_{k}}^{\Lambda_{1},[k], m_{\lambda_{1}}, \tau^{\prime}} A_{a_{1} \lambda_{1}}^{\dagger} A_{a_{2} \lambda_{2}}^{\dagger} \cdots A_{a_{k} \lambda_{k}}^{\dagger}|0\rangle
$$

The first Clebsch's are for the symmetric traceless of $S O(4)$ which are precisely the representations we discussed under (132). The second Clebsch have been discussed before in Section 2. They decompose the $\operatorname{Sym}\left(V_{H}^{\otimes k}\right)$ into irreps. of $\Lambda_{1}$ of $S_{n}$. When we form gauge invariant operators as in Section 6 there are constraints relating $\Lambda_{1}$ to the $U(N)$ Young diagram $R$ which organises the traces. This representation $R$ in the half-BPS case allows a map to the type of giant [8]. Young diagrams with a few (order 1) long (order $N$ for example) columns map to sphere giants. Those with a few long rows map to AdS giants. Non-abelian worldvolume symmetries emerge when we have rows or columns of equal length. This map also works for open string excitations and there are elegant tests involving the counting of states which are sensitive to the presence of non-abelian symmetries [10, 11].

Consider Young diagrams of the form $R=[n]$ which correspond to single AdS giants of angular momentum $n$. Recall that the gauge invariant operators are labelled by $R, \Lambda_{n+k}, M, \Lambda_{1}, \tau, \tau_{\Lambda_{1}, R} . \quad R$ is a $U(N)$ irrep. $\Lambda_{n+k}$ is the lowest weight of the $S L(2)$ which is completely determined by the excitation energy $l$. $M$ labels states in $\Lambda_{n+k}$. $\Lambda_{1}$ is an irrep. of $S_{n}$. $\tau$ runs over the multiplicity of $\Lambda_{1}$ in the symmetric tensor product of the hook representation. $\tau_{\Lambda_{1}, R}$ runs from 1 to $C\left(R, R, \Lambda_{1}\right)$. For fixed $R$ the multiplicity of LWS is

$$
\sum_{\Lambda_{1}} C\left(R, R, \Lambda_{1}\right) \operatorname{Mult}\left(\operatorname{Sym}\left(V_{H}^{\otimes k}\right), \Lambda_{1}\right)
$$

By summing over states for fixed $R$ we can get excited states of a fixed type of giant worldvolume. In particular we are interested in $R=[n]$. The inner tensor product of $R$ with itself only contains the identity rep. $\Lambda_{1}=[n]$. So the number of lowest weights at level $k$ is just the multiplicity of $[n]$ in the symmetric tensor product of the hook. We have a generating function for this derived in Section 3. The generating function including the descendants, is (using (47) or (51))

$$
\frac{1}{(1-q)\left(1-q^{2}\right)\left(1-q^{3}\right) \cdots\left(1-q^{n}\right)}
$$

The coefficient of $q^{k}$ is the number of partitions of $k$ with no part bigger than $n$. Note that $n$ is the number of boxes in the Young diagram describing the giant. For the semiclassical approximation of giant brane worldvolume to be valid, this is of order $N^{\alpha}$ (for $\alpha$ close to $1), k$ is the excitation on the brane worldvolume, which we are treating in a linearized approximation, so we certainly want that to be small compared to $n$. When $k$ is smaller 
than $n$, the above just counts unrestricted partitions of $k$. This matches the counting of Fock space states in (136).

Hence, in the regime of interest, where $k$ is much bigger than one (so we can expect GKP 38. type arguments to be valid) but smaller than the energy of the brane, the above counting of partitions of $k$ is exactly what we are getting from quantizing a class of vibrations of the AdS giant. Using this emergence of Fock space structures from the counting of states in the tensor product of $\operatorname{Sym}\left(V_{H}^{\otimes k}\right)$ we therefore find the correct counting of gauge theory operators which correspond to states of the form (131) and (132) with energy $L_{0}=n+k$ and with a single spin $k$ in the case (131) or with $S O(4)$ representation $[k]$ for (132).

In fact we can also see where the six different species of oscillations could come from. In the above discussion we have been considering BPS giants built from Schur polynomials of $X=X_{1}+i X_{2}$ and then perturbed by replacing $X$ with derivatives $P_{\lambda}$ acting on $X$, of the form $P_{\lambda}^{*} X$. We could also consider powers of $P_{\lambda}$ acting on $X_{i}$ (with $i=3,4,5,6$ ) replacing the $X$. And finally we can consider powers of $P_{\lambda}$ acting on $X^{\dagger}$ as the impurities. So in all we have six types of impurities $P_{\lambda}^{*} X, P_{\lambda}^{*} X^{\dagger}, P_{\lambda}^{*} X_{i}$. These correspond to six sets of gauge invariant operators matching states with the right energies of the form (131), which come, in the spacetime worldvolume analysis to exciting quanta of $\delta r, \delta v_{m}, \delta \phi$ excitations. Given the simplicity of $\omega_{-}$we would expect that they correspond to the simplest construction in gauge theory, namely using $P_{\lambda}^{*} X$ impurities, which they match precisely in energy. If we consider the states in (133) and restrict to the case where all the impurities are of the same type and the $S O(4)$ representation is $[k]$ with the energy being $E=n+k$, then the above discussion extends easily to give the corresponding gauge theory duals. A complete account of the case with mixed impurities will be left for the future.

\subsection{Comments}

There are many interesting extensions of the above discussion which could be considered. We have chosen the simplest $R$ of the form $[n]$ which correspond to AdS giants. If we consider $R=\left[n_{1}, n_{2}\right]$ and sum over $\Lambda_{1}$ as in (137) this should correspond to excitations in spacetime of multiple-giants described by a $U(2)$ (if $\left.n_{1}=n_{2}\right)$ or $U(1) \times U(1)$ (if $n_{1} \neq n_{2}$ ) worldvolume DBI gauge theory. A similar simple counting of states holds true for excitations of S-giants [22]. They will be associated to spherical harmonics of an $S O(4)$ in the $S O(6)$. So we expect that excitations in the gauge theory from the $S O(6)$ sector should also have this kind of free field counting in an appropriate large angular momentum limit. The $S O(4) \subset S O(4,2)$ excitations considered in (136) also exist for $R=\left[1^{n}\right]$. They should correspond to excitations of sphere giants, but it is not obvious to us how a Fock space structure emerges from considering their motions in the transverse AdS. It will be interesting to clarify this puzzle.

Note that we are making here a comparison between zero coupling in Yang Mills to spacetime calculations dual to strong coupling Yang Mills. This works best for large angular momenta where $l$ is large so that the frequencies can all be approximated by $\omega=l$, but smaller than $n$ which is the large angular momentum of the giant. This gives a different 
context of excitations of giant gravitons, where the basic idea of large quantum numbers allowing strong to weak coupling comparisons [38] continues to apply. Here the parameters $N, k, n$ are all large.

There have been earlier discussions of supersymmetric states obtained from the quantization of moduli spaces of giants and the comparison with gauge theory counting [39, 40, 41, 42, In the discussion above we have been interested in all the excitations in the free theory of a given half-BPS giant. A subset of these will be supersymmetric but a lot of the states will be non-supersymmetric. We expect that, in analogy with discussions of semiclassical strings [38, 37] appropriate limits of large quantum numbers can be used to compare nonsupersymmetric states. The new technical ingredient in the above treatment is the use of a diagonal basis of gauge theory operators at finite $N$, where the label $R$ allows the identification of the giant in question, and additional global symmetry labels help the map to objects in spacetime. The use of symmetric group data in organising the multiplicities of states for fixed $R$ and fixed global symmetry quantum numbers shows the emergence, in the limit of large $n$, of Fock space counting from properties of symmetric group decompositions such as $\operatorname{Sym}\left(V_{H}^{\otimes n}\right)$. At finite $n$ we have a cut-off Fock space.

\section{One-loop mixing in $\mathcal{N}=4$}

In [43] the one-loop mixing of the Clebsch-Gordan basis introduced in [15] for the $G=U(2)$ sector of $\mathcal{N}=4$ super Yang-Mills was analysed. These operators only mix if the $U(N)$ representations specifying their multi-trace structures are related by the repositioning of a single box of the Young diagram. Here we find the same result for the full $P S U(2,2 \mid 4)$ sector, using our general characterisation of multi-trace operators with arbitrary global symmetry.

The complete one-loop non-planar dilatation operator is given by [26]

$$
D(g)=D_{0}-\frac{g_{Y M}^{2}}{8 \pi^{2}} H+\mathcal{O}\left(g_{Y M}^{3}\right)
$$

where

$$
H=\sum_{j=0}^{\infty} h(j)\left(P_{j}\right)_{C D}^{A B}: \operatorname{tr}\left(\left[W_{A}, \tilde{W}^{C}\right]\left[W_{B}, \tilde{W}^{D}\right]\right):
$$

$\left(\tilde{W}^{C}\right)_{j}^{i}$ is the derivative $\frac{d}{d\left(W_{C}\right)_{i}^{j}} . \quad h(j) \equiv \sum_{k=1}^{j} \frac{1}{k}$ are the harmonic numbers and $P_{j}$ is the projector for $V_{F} \otimes V_{F}=\oplus_{j} V_{j}$. For $S L(2)$ and $P S U(2,2 \mid 4) V_{j}$ appears with unit multiplicity in $V_{F}^{\otimes 2}$ (cf. (43) where $\left.m(j, 2)=1\right) \sqrt{3}$. The dilatation operator separates out $V_{F}^{\otimes 2}$ in $V_{F}^{\otimes n}$ and then projects onto it with the factors in (140).

The action of the dilatation operator has been analysed in the planar limit for single traces using the Bethe Ansatz (see for example [44] 45]). In the non-planar limit multi-trace

\footnotetext{
${ }^{3}$ In the $S L(2) \times S_{2}$ decomposition of $V_{F}^{\otimes 2}$, the symmetric representation $V_{[2]}^{S_{2}}$ appears with even $j$ and the antisymmetric $V_{[1,1]}^{S_{2}}$ with odd $j$.
} 
operators can join and split [46]. We will find that the mixing is neatly constrained if we organise the multi-trace operators using $U(N)$ representations as we have in (109).

The action of $H$ on $\operatorname{tr}\left(\alpha W_{m_{1}} \cdots W_{m_{n}}\right)$ is compactly written by introducing an extra index, tracing in $V_{N}^{n+1}$ rather than $V_{N}^{n}$. The extra index encodes awkward contractions in the action of the dilatation operator.

$$
\begin{aligned}
& : \operatorname{tr}\left(\left[W_{A}, \tilde{W}^{C}\right]\left[W_{B}, \tilde{W}^{D}\right]\right): \operatorname{tr}\left(\alpha W_{m_{1}} \cdots W_{m_{n}}\right)= \\
& \frac{1}{(n-2) !} \sum_{\sigma \in S_{n}} \delta_{m_{\sigma(n-1)}}^{C} \delta_{m_{\sigma(n)}}^{D} \sum_{\rho_{1}, \rho_{2} \in S_{n+1}} f\left(\rho_{1}, \rho_{2}\right) \operatorname{tr}_{n+1}\left(\rho_{1} \sigma^{-1} \alpha \sigma \rho_{2} W_{m_{\sigma(1)}} \cdots W_{m_{\sigma(n-2)}} W_{A} W_{B} \mathbb{I}_{N}\right)
\end{aligned}
$$

$\mathbb{I}_{N}$ is the $N \times N$ identity matrix. $f\left(\rho_{1}, \rho_{2}\right)$ is only non-zero on the $S_{3}$ subgroup of $S_{n+1}$ that permutes the $n-1$ and $n$ indices, where the derivatives act, and the new $n+1$ index. Its non-zero values give the four terms of the commutators in (140).

$$
\begin{aligned}
f((n-1, n),(n, n+1)) & =1 \\
f((n-1, n+1),(n, n+1)) & =-1 \\
f((n, n+1),(n-1, n+1)) & =-1 \\
f((n, n+1),(n-1, n)) & =1
\end{aligned}
$$

If we introduce the projector we find

$$
\begin{aligned}
& \sum_{j=0}^{\infty} h(j)\left(P_{j}\right)_{C D}^{A B}: \operatorname{tr}\left(\left[W_{A}, \tilde{W}^{C}\right]\left[W_{B}, \tilde{W}^{D}\right]\right): \operatorname{tr}\left(\alpha W_{m_{1}} \cdots W_{m_{n}}\right)=\sum_{\rho_{1}, \rho_{2} \in S_{n+1}} f\left(\rho_{1}, \rho_{2}\right) \\
& \frac{1}{(n-2) !} \sum_{\sigma \in S_{n}} \sum_{j=0}^{\infty} h(j) \operatorname{tr}_{n+1}\left(\rho_{1} \sigma^{-1} \alpha \sigma \rho_{2} W_{m_{\sigma(1)}} \cdots W_{m_{\sigma(n-2)}} P_{j}\left(W_{m_{\sigma(n-1)}} W_{m_{\sigma(n)}}\right) \mathbb{I}_{N}\right)
\end{aligned}
$$

Now consider the action on our gauge-invariant operator (109)

$$
\begin{aligned}
& H \mathcal{O}^{\Lambda, M_{\Lambda}, \Lambda_{1}, \tau, R, \tau_{\Lambda_{1}, R}}=\frac{1}{(n-2) !} \sum_{\rho_{1}, \rho_{2} \in S_{n+1}} f\left(\rho_{1}, \rho_{2}\right) C^{\tau_{\Lambda_{1}, R}{ }_{m_{\Lambda_{1}}}^{R} \underset{j}{R} \sum_{\alpha \in S_{n}}} D_{i j}^{R}(\alpha) \\
& \sum_{j=0}^{\infty} h(j) C_{\Lambda, M_{\Lambda}, \Lambda_{1}, m_{\Lambda_{1}}, \tau}^{\vec{m}} \operatorname{tr}_{n+1}\left(\rho_{1} \alpha \rho_{2} W_{m_{1}} \cdots W_{m_{n-2}} P_{j}\left(W_{m_{n-1}} W_{m_{n}}\right) \mathbb{I}_{N}\right)
\end{aligned}
$$

Here, using properties of our operators, all the $\sigma$ actions cancel.

To encapsulate the action of the projector we rewrite the covariant decomposition of $V_{F}^{\otimes n}$ in terms of $V_{F}^{\otimes n-2} \otimes V_{F}^{\otimes 2}$. We unclutter the notation by defining $|\boldsymbol{\Lambda}\rangle \equiv\left|\Lambda, M_{\Lambda}, \lambda, m_{\lambda}, \tau\right\rangle$ for the covariant basis.

$$
\begin{aligned}
|\boldsymbol{\Lambda}\rangle & =\sum_{\vec{m}} C_{\boldsymbol{\Lambda}}^{\vec{m}} \sum_{\boldsymbol{\Lambda}^{n-2}, \boldsymbol{\Lambda}^{2}} C_{\vec{m}^{n-2}}^{\boldsymbol{\Lambda}^{n-2}} C_{\vec{m}^{2}}^{\boldsymbol{\Lambda}^{2}}\left|\boldsymbol{\Lambda}^{n-2}\right\rangle \otimes\left|\boldsymbol{\Lambda}^{2}\right\rangle \\
& =\sum_{\boldsymbol{\Lambda}^{n-2}, \boldsymbol{\Lambda}^{2}}\left\langle\boldsymbol{\Lambda}^{n-2}, \boldsymbol{\Lambda}^{2} \mid \boldsymbol{\Lambda}\right\rangle\left|\boldsymbol{\Lambda}^{n-2}, \boldsymbol{\Lambda}^{2}\right\rangle
\end{aligned}
$$


$\left|\boldsymbol{\Lambda}^{n-2}\right\rangle$ lives in $V_{F}^{\otimes n-2}$ while $\left|\boldsymbol{\Lambda}^{2}\right\rangle$ lives in $V_{F}^{\otimes 2} . \vec{m}^{n-2}=\left(m_{1}, \ldots, m_{n-2}\right)$ and $\vec{m}^{2}=\left(m_{n-1}, m_{n}\right)$. The projector $P_{j}$ in (142) projects onto $\Lambda^{2}=j$. The one-loop two-point function is then

$$
\begin{aligned}
& \left\langle\left(\mathcal{O}^{\dagger}\right)^{\Lambda^{\prime}, M_{\Lambda^{\prime}}^{\prime}, \Lambda_{1}^{\prime}, \tau^{\prime}, R^{\prime}, \tau_{\Lambda_{1}^{\prime}, R^{\prime}}^{\prime}} H \mathcal{O}^{\Lambda, M_{\Lambda}, \Lambda_{1}, \tau, R, \tau_{\Lambda_{1}, R}}\right\rangle
\end{aligned}
$$

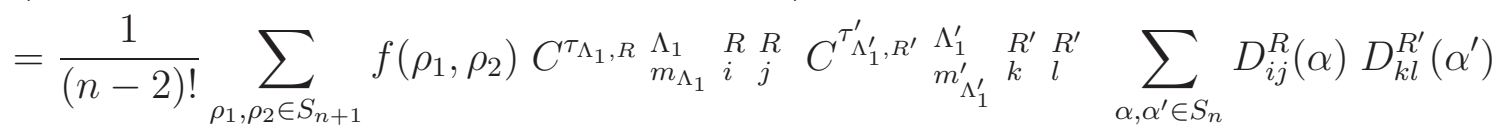

$$
\begin{aligned}
& \sum_{\boldsymbol{\Lambda}^{n-2}, \boldsymbol{\Lambda}^{2}=j} h(j)\left\langle\boldsymbol{\Lambda}^{\prime} \mid \boldsymbol{\Lambda}^{n-2}, \boldsymbol{\Lambda}^{2}\right\rangle\left\langle\boldsymbol{\Lambda}^{n-2}, \boldsymbol{\Lambda}^{2} \mid \boldsymbol{\Lambda}\right\rangle \operatorname{tr}_{n+1}\left(\rho_{1} \alpha \rho_{2} \alpha^{\prime} \mathbb{I}_{N}^{n+1}\right)
\end{aligned}
$$

The trace can be expressed as a sum over $(n+1)$-box representations $T$ of $S_{n+1}$ and $U(N)$ with at most $N$ rows.

$$
\operatorname{tr}_{n+1}\left(\rho_{1} \alpha \rho_{2} \alpha^{\prime} \mathbb{I}_{N}^{n+1}\right)=\sum_{T \vdash n+1} \chi_{T}\left(\rho_{1} \alpha \rho_{2} \alpha^{\prime}\right) \operatorname{Dim} T
$$

The $\alpha$ and $\alpha^{\prime}$ sums in (144) force $T$ to reduce to both $R$ and $R^{\prime}$ for the $S_{n}$ subgroup of $S_{n+1}$. Since $T$ reduces on its $S_{n}$ subgroup to those Young diagrams with a single box removed from $T, R$ and $R^{\prime}$ must be related by the repositioning of a single box for this one-loop two-point function not to vanish. This analysis is pursued in more detail in [43.

The one-loop non-planar mixing of this complete basis of multi-trace operators is therefore highly constrained. Although the operators are not diagonal at one-loop, their very limited mixing suggests they are close to the eigenstates. It would be particularly interesting to find the sixteenth-BPS operators at one loop and gain an understanding of the counting of black hole entropy, along the lines of [47, 42].

\section{Summary and Outlook}

We have given a general construction of diagonal gauge invariant operators for a $U(N)$ gauge theory with global symmetry $G$ in the free field limit. The Clebsch-Gordan coefficients for the $G \times S_{n}$ decomposition of $V_{F}^{\otimes n}$, where $V_{F}$ is a fundamental representation of $G$, play a crucial role. We have exploited the result of [15] which showed that once the covariant correlators are brought to a standard form, which we have called the "canonical covariant form" then the gauge invariant diagonalisation follows using Clebsch-Gordan coefficients of $S_{n}$. The $G \times S_{n}$ decomposition contains representation labels $\Lambda, \Lambda_{1}$ of $G$ and $S_{n}$ respectively. We showed in this paper how to use the corresponding Clebsch-Gordan coefficients, obeying standard Clebsch orthogonality properties (69), to construct operators with correlators of the canonical covariant form (77).

The construction of gauge invariant objects uses a representation label $R$ corresponding to $U(N)$ and its Schur-Weyl dual $S_{n}$. This label appears in the simplest set-up in the half-BPS sector [8], and is interpreted in terms of giant gravitons. The final step of going 
from canonical covariant form to gauge invariant diagonal form uses the Clebsch-Gordan coefficients for the $S_{n}$ inner tensor product $R \otimes R \rightarrow \Lambda_{1}$. We showed that the construction of a diagonal basis of gauge invariant operators matches the counting of gauge invariant operators done using Matrix Model techniques [21].

As special cases we have considered $G=U(M), S L(2), S O(6)$ which are relevant to specific sectors of $\mathcal{N}=4 \mathrm{SYM}$ theory. In the case of $U(M)$ we have shown that the multimatrix diagonalisation result of [15] contains a formula for the Clebsch-problem of $U(M)$ decomposition of $V_{M}^{\otimes n}$ in terms of the symmetric group data of branching coefficients. For $S L(2)$ we have shown how the $S L(2) \times S_{n}$ Clebsch-problem for the $n$-fold tensor product of the discrete series representation spanned by $X, \partial X, \partial^{2} X \ldots$ can be solved by considering one energy level at a time, labelled by $k$, the total number of derivatives involved in the $n$-fold tensor product. The total number of lowest weight states appearing at fixed $k$ can be neatly described in terms of oscillator constructions of $S L(2)$. This leads to a mapping of the problem of diagonalising the multiplicity of LWS at fixed $k$ into a problem involving the $S_{n} \times S_{k}$ decomposition of the $k$-fold tensor power of a hook representation of $S_{n}$ of dimension $n-1$. This $S_{n} \times S_{k}$ problem has some surprising Fock space structures in the large $n, k$ limit. These structures have been used to identify gauge invariant operators with energies and multiplicities matching those appearing in earlier work on the excitations of giant gravitons computed from the point of view of a worldvolume analysis [22].

We expect that further investigations on the diagonalisation of gauge invariant operators will allow more detailed comparisons between excitations of giant gravitons in spacetime and gauge theory operators. Comparisons going beyond free fields in gauge theory and beyond the leading semiclassical approximations in giant gravitons will also be instructive. The formula for the 1-loop dilatation operator acting on our basis, given in Section (8), is a step in this direction.

Acknowledgements We thank Sumit Das, Robert de Mello Koch, Yusuke Kimura, David Turton for discussions. SR is supported by an STFC Advanced Fellowship. PJH is supported by an EPSRC Standard Research Grant EP/C544250/1. TWB is on an STFC studentship.

\section{A Clebsch-Gordan decomposition for $V_{H}^{\otimes k}$}

We will collect several useful facts about the decomposition into $S_{n} \times S_{k}$ representations of the $k$-fold tensor product $V_{H}^{\otimes k}$ of the hook representation $V_{H}$ of $S_{n}$ associated with the Young diagram $[n-1,1]$. 


\section{A.1 Multiplicities from characters}

The $k$-fold tensor product decomposes as follows

$$
V_{H}^{\otimes k}=\bigoplus_{\Lambda_{1}, \Lambda_{2}} V_{\Lambda_{1}} \otimes V_{\Lambda_{2}} \otimes V_{\Lambda_{1}, \Lambda_{2}}
$$

Here $V_{\Lambda_{1}}$ is an irrep of $S_{n}, V_{\Lambda_{2}}$ is an irrep. of $S_{k}$, and $V_{\Lambda_{1}, \Lambda_{2}}$ is an irrep of $\operatorname{Com}\left(S_{n} \times S_{k}\right)$, the algebra commuting with $S_{n} \times S_{k}$ in the $V_{H}^{\otimes k}$.

The dimensions $d_{\Lambda_{1}, \Lambda_{2}}$ of $V_{\Lambda_{1}, \Lambda_{2}}$ appear in the oscillator construction of LWS (lowest weight states) in the tensor products of the fundamental $S L(2)$ representation. We can calculate these dimensions using characters of $S_{n}$ and $S_{k}$.

$$
d_{\Lambda_{1}, \Lambda_{2}}=t r_{V_{H}^{\otimes k}}\left(\frac{P_{\Lambda_{1}}}{d_{\Lambda_{1}}} \otimes \frac{P_{\Lambda_{2}}}{d_{\Lambda_{2}}}\right)
$$

The projectors $P_{\Lambda_{1}}, P_{\Lambda_{2}}$ are given by

$$
\frac{P_{\Lambda_{1}}}{d_{\Lambda_{1}}}=\frac{1}{n !} \sum_{\sigma \in S_{n}} \chi_{\Lambda_{1}}(\sigma) \sigma
$$

and

$$
\frac{P_{\Lambda_{2}}}{d_{\Lambda_{2}}}=\frac{1}{k !} \sum_{\tau \in S_{k}} \chi_{\Lambda_{2}}(\tau) \tau
$$

Hence we can write

$$
\begin{aligned}
& d_{\Lambda_{1}, \Lambda_{2}}=\frac{1}{k !} \sum_{\tau \in S_{k}} \chi_{\Lambda_{2}}(\tau) \frac{1}{n !} \sum_{\sigma \in S_{n}} \chi_{\Lambda_{1}}(\sigma) \operatorname{tr}_{V_{H}^{\otimes k}}(\tau \otimes \sigma) \\
& =\frac{1}{k !} \sum_{\tau \in S_{k}} \chi_{\Lambda_{2}}(\tau) \frac{1}{n !} \sum_{\sigma \in S_{n}} \chi_{\Lambda_{1}}(\sigma) \prod_{i}\left(\operatorname{tr}_{V_{H}}\left(\sigma^{i}\right)\right)^{c_{i}(\tau)}
\end{aligned}
$$

For computer code to calculate this multiplicity see Section C. Here $c_{i}(\tau)$ is the number of cycles of length $i$ in the permutation $\tau$. To see this note that

$$
\begin{aligned}
& \left\langle a_{1} . . a_{k}|\sigma| a_{1} . . a_{k}\right\rangle=\left\langle a_{1} . . a_{k} \mid \sigma\left(a_{1}\right) \cdots \sigma\left(a_{k}\right)\right\rangle \\
& \left\langle a_{1} . . a_{k}|\tau| a_{1} . . a_{k}\right\rangle=\left\langle a_{1} . . a_{k} \mid a_{\tau(1)} \cdots a_{\tau(k)}\right\rangle \\
& \left\langle a_{1}|\sigma| a_{2}\right\rangle=D_{a_{1} a_{2}}^{H}(\sigma)
\end{aligned}
$$

\section{A.2 Clebsch-Gordan coefficients}

For the Clebsch-decomposition of $V_{H}^{\otimes k}$ into $\Lambda_{1}\left(S_{n}\right) \otimes \Lambda_{2}\left(S_{k}\right)$, the first thing we need is the multiplicities. We also need in Section 2 the properties of the Clebsch-Gordan coefficients for the case $\Lambda_{2}=[k]$. Here we state some general properties valid for any $\Lambda_{2}$.

For the basic Clebsch problem of coupling a pair of irreps to a third $R \otimes S \rightarrow T$ we have previously used formulae of the type $D D D=C C$ and $D D C=D C$ derived for example in 
[48] 4 . These were derived by inserting complete sets of states etc. Similar techniques lead to similar equations, which allow us, given the matrix elements of $S_{n}$ irreps., to compute the Clebsch.

The following is the analog of $D D C=D C$

$$
\begin{aligned}
& \sum_{a_{1}, \cdots, a_{k}} D_{b_{1} a_{\tau(1)}}^{H}(\sigma) D_{b_{2} a_{\tau(2)}}^{H}(\sigma) \cdots D_{b_{k} a_{\tau(k)}}^{H}(\sigma) C_{a_{1} \ldots a_{k}}^{\Lambda_{1}, \Lambda_{2}, m_{\Lambda_{1}}, m_{\Lambda_{2}} ; \tau_{\Lambda_{1}, \Lambda_{2}}} \\
= & C_{b_{1} \cdots b_{k}}^{\Lambda_{1}, \Lambda_{2}, m_{\Lambda_{1}}^{\prime}, m_{\Lambda_{2}}^{\prime} ; \tau_{\Lambda_{1}, \Lambda_{2}}} D_{m_{\Lambda_{1}}^{\prime} m_{\Lambda_{1}}}^{\Lambda_{1}}(\sigma) D_{m_{\Lambda_{2}}^{\prime} m_{\Lambda_{2}}}^{\Lambda_{2}}(\tau)
\end{aligned}
$$

The following is the analog of $D D D=C C$.

$$
\begin{aligned}
& \sum_{\sigma \in S_{n}} \sum_{\tau \in S_{k}} D_{m_{\Lambda_{1}}, m_{\Lambda_{1}}^{\prime}}^{\Lambda_{1}}(\sigma) D_{m_{\Lambda_{2}}, m_{\Lambda_{2}}^{\prime}}^{\Lambda_{2}}(\tau) \quad D_{a_{1} b_{\tau(1)}}^{H}(\sigma) \cdots D_{a_{k} b_{\tau(k)}}^{H}(\sigma) \\
& =\sum_{\tau_{\Lambda_{1}, \Lambda_{2}}} C_{b_{1} \ldots b_{k}}^{\tau_{\Lambda_{1}, \Lambda_{2}, \Lambda_{1}, \Lambda_{2}, m_{\Lambda_{1}}, m_{\Lambda_{2}}}} C_{a_{1} \ldots a_{k}}^{\tau_{\Lambda_{1}, \Lambda_{2}, \Lambda_{1}, \Lambda_{2}, m_{\Lambda_{1}}^{\prime}, m_{\Lambda_{2}}^{\prime}}^{\prime}}
\end{aligned}
$$

\section{A.3 Symmetrised Clebsch from ordinary Clebsch}

Now we specialise to give the properties for $\Lambda_{2}=[k]$ which involve the symmetrised Clebsch. The symmetrised Clebsch-Gordan coefficients with the properties used in Section 2 can be obtained from the Clebsch-Gordans for ordinary tensor products $V_{H}^{\otimes k}$. Consider states $\left|a_{1}, a_{2} \cdots a_{k}\right\rangle$ in the tensor product. The action on the tensor product is

$$
\sigma\left|a_{1}, \cdots, a_{k}\right\rangle=D_{b_{1} a_{1}}^{H}(\sigma) \cdots D_{b_{k} a_{k}}^{H}(\sigma)\left|b_{1}, \cdots, b_{k}\right\rangle
$$

This action of $\sigma \in S_{n}$ commutes with $S_{k}$ permutations which act as

$$
\alpha\left|a_{1}, \cdots, a_{k}\right\rangle=\left|a_{\alpha(1)}, \cdots, a_{\alpha(k)}\right\rangle
$$

The symmetric subspace of $V_{H}^{\otimes k}$ is isomorphic to the space of $k$ oscillators $A_{a_{1}} \cdots A_{a_{k}}$. We can identify $A_{a_{1}} \cdots A_{a_{k}}$ with

$$
P_{\text {sym }}\left|a_{1}, \cdots, a_{k}\right\rangle
$$

where the projector is $P_{\text {sym }}=\frac{1}{k !} \sum_{\alpha \in S_{k}} \alpha$ acting on $V_{H}^{\otimes k}$. The usual Clebsch decomposition gives the transformation matrix from the tensor product basis to a basis of irreps. $\left|\Lambda_{1}, i, \tau\right\rangle$ where $\tau$ is a multiplicity index and $i$ is runs over the dimension $d_{\Lambda_{1}}$. Since $P_{\text {sym }}$ commutes with $S_{n}$, its eigenvalues are constant on irreps, and since it is a projector they are 1 or 0 . We can define the symmetric irreps $\Lambda_{1}, i$ to be the set left invariant by $P_{\text {sym }}$. So we have

$$
P_{\text {sym }}\left|\Lambda_{1}, i, \tau\right\rangle=\left|\Lambda_{1}, i, \tau\right\rangle
$$

\footnotetext{
${ }^{4}$ A minor notational point is that we are using the symbol $C$ for Clebsch in this paper rather than $S$ as in [15] and 48.
} 
The Clebsch-Gordans restricted to the symmetric irreps are the symmetrised Clebsch-Gordans

$$
\begin{aligned}
& C_{a_{1} \cdots a_{k}}^{\Lambda_{1}, i} \equiv\left\langle\Lambda_{1}, i, \tau \mid a_{1} \cdots a_{k}\right\rangle \\
& =\left\langle\Lambda_{1}, i, \tau\left|P_{\text {sym }}\right| a_{1} \cdots a_{k}\right\rangle
\end{aligned}
$$

If we permute the vectors in the tensor product we have

$$
\begin{aligned}
C_{a_{\alpha(1)} \cdots a_{\alpha(k)}}^{\Lambda_{1}, i, \tau} & =\left\langle\Lambda_{1}, i, \tau\left|P_{\text {sym }} \alpha\right| a_{1} \cdots a_{k}\right\rangle \\
& =\left\langle\Lambda_{1}, i, \tau\left|P_{\text {sym }}\right| a_{1} \cdots a_{k}\right\rangle \\
& =C_{a_{1} \cdots a_{k}}^{\Lambda_{1}, i, \tau}
\end{aligned}
$$

The $\alpha$ can be absorbed in the redefinition of the summation over permutations in $P_{\text {sym }}$. This gives the desired symmetry of the symmetrised Clebsch (34).

Orthogonality (36) follows from the restriction of the usual orthogonality to the symmetric subspace. The identity (35) follows by considering

$$
\begin{aligned}
\left\langle\Lambda_{1}, i, \tau|\sigma| b_{1}, \cdots, b_{k}\right\rangle & =\left\langle\Lambda_{1}, i, \tau\left|P_{\text {sym }} \sigma\right| b_{1}, \cdots, b_{k}\right\rangle \\
& =\left\langle\Lambda_{1}, i, \tau\left|\sigma P_{\text {sym }}\right| b_{1}, \cdots, b_{k}\right\rangle
\end{aligned}
$$

and inserting on the left of $\sigma$ a complete set of tensor product states or on the right of $\sigma$ a complete set of symmetric irrep states.

\section{A.4 Symmetrised Clebsch multiplicities}

We give some examples of the symmetrised Clebsch multiplicities for $V_{H}=[n-1,1]$. The tensor product of $V_{H} \otimes V_{H}$ is decomposed into irreps as $[n]+[n-1,1]+[n-2,2]+\left[n-2,1^{2}\right]$. It can be checked that their dimensions add up to $(n-1)^{2}$. If we drop the last we get the dimensions adding to $\frac{n(n-1)}{2}$ as expected form the symmetric product. So we have

$$
\operatorname{Sym}\left(V_{H} \otimes V_{H}\right)=[n]+[n-1,1]+[n-2,2]
$$

To get $\operatorname{Sym}\left(V_{H} \otimes V_{H} \otimes V_{H}\right)$ we first take the above symmetric tensor product and then a further tensor product with $[n-1,1]$. We need the tensor product

$$
[n-2,2] \otimes[n-1,1]=[n-1,1]+[n-2,2]+[n-2,1,1]+[n-3,3]+[n-3,2,1]
$$

The dimensions add correctly. Then we need to implement the symmetric projection. One finds that the following gives the correct dimension counting

$$
\operatorname{Sym}\left(V_{H} \otimes V_{H} \otimes V_{H}\right)=[n]+2[n-1,1]+[n-2,2]+[n-2,1,1]+[n-3,3]
$$

So the representation $[n-3,2,1]$ together with one of the two reps $[n-2,1,1]$, one of the two reps $[n-2,2]$, and one of the three reps $[n-1,1]$ have been projected out from $\operatorname{Sym}\left(V_{H} \otimes V_{H}\right) \otimes V_{H}$. 


\section{B Action of $S_{n}$ on $A_{a}^{\dagger}$ in detail}

The orthogonal basis of $A_{a}^{\dagger}$ which we used in Section 2 has the property that $S_{n}$ acts on it via the standard Young-Yamanouchi orthogonal basis of the $[n-1,1]$ hook representation (as given for example in [48]). If $s_{i}=(i, i+1)$ are the 2 -cycle permutations that generate $S_{n}$ then we have

$$
\begin{aligned}
s_{i} A_{a}^{\dagger} & =A_{a}^{\dagger} \quad \text { for } i \leq a-1 \text { and } i \geq a+2 \\
s_{i+1} A_{i}^{\dagger} & =\frac{1}{i+1} A_{i}^{\dagger}+\frac{\sqrt{i(i+2)}}{i+1} A_{i+1}^{\dagger} \\
s_{i+1} A_{i+1}^{\dagger} & =\frac{\sqrt{i(i+2)}}{i+1} A_{i}^{\dagger}-\frac{1}{i+1} A_{i+1}^{\dagger}
\end{aligned}
$$

We can identify the representation of $S_{n}$ formed by the $A_{a}$ using general arguments. It is easy to see that there is no invariant vector under $S_{n}$, and that there is one invariant vector under $S_{n-1}$ (namely $A_{n-1}$ ). The only irreducible representations of $S_{n}$ which contain the invariant of $S_{n-1}$ are $[n]$ and $[n-1,1]$. Having ruled out the symmetric irrep. [n], the $(n-1)$ dimensional representation formed by the $A_{a}$ can only be the irreducible $[n-1,1]$. More directly we can use the construction of the orthogonal representing matrices given in [48], which uses branching arguments.

\section{Code}

Code written to calculate the various multiplicities discussed here is available under the GNU General Public Licence at http://www.nworbmot.org/code/. It is written in python for use with the SAGE open source computer algebra system.

\section{References}

[1] J. M. Maldacena, "The large N limit of superconformal field theories and supergravity," Adv. Theor. Math. Phys. 2 (1998) 231 [Int. J. Theor. Phys. 38 (1999) 1113] [arXiv:hepth/9711200].

[2] S. S. Gubser, I. R. Klebanov and A. M. Polyakov, "Gauge theory correlators from noncritical string theory," Phys. Lett. B 428 (1998) 105 [arXiv:hep-th/9802109].

[3] E. Witten, "Anti-de Sitter space and holography," Adv. Theor. Math. Phys. 2 (1998) 253 [arXiv:hep-th/9802150].

[4] J. M. Maldacena and A. Strominger, "AdS(3) black holes and a stringy exclusion principle," JHEP 9812 (1998) 005 [arXiv:hep-th/9804085]. 
[5] J. McGreevy, L. Susskind and N. Toumbas, "Invasion of the giant gravitons from anti-de Sitter space," JHEP 0006 (2000) 008 [arXiv:hep-th/0003075].

[6] M. T. Grisaru, R. C. Myers and O. Tafjord, "SUSY and Goliath," JHEP 0008, 040 (2000) [arXiv:hep-th/0008015]; A. Hashimoto, S. Hirano and N. Itzhaki, "Large branes in AdS and their field theory dual," JHEP 0008, 051 (2000) [arXiv:hep-th/0008016]; V. Balasubramanian, M. Berkooz, A. Naqvi and M. J. Strassler, "Giant gravitons in conformal field theory," JHEP 0204 (2002) 034 [arXiv:hep-th/0107119].

[7] V. Balasubramanian, M. Berkooz, A. Naqvi and M. J. Strassler, "Giant gravitons in conformal field theory," JHEP 0204 (2002) 034 [arXiv:hep-th/0107119].

[8] S. Corley, A. Jevicki and S. Ramgoolam, "Exact correlators of giant gravitons from dual N = 4 SYM theory," Adv. Theor. Math. Phys. 5 (2002) 809 [arXiv:hep-th/0111222].

[9] D. Berenstein, "A toy model for the AdS/CFT correspondence," JHEP 0407 (2004) 018 [arXiv:hep-th/0403110].

[10] V. Balasubramanian, D. Berenstein, B. Feng and M. x. Huang, "D-branes in Yang-Mills theory and emergent gauge symmetry," JHEP 0503 (2005) 006 [arXiv:hep-th/0411205].

[11] R. de Mello Koch, J. Smolic and M. Smolic, "Giant Gravitons - with Strings Attached (I)," JHEP 0706 (2007) 074 [arXiv:hep-th/0701066]; R. de Mello Koch, J. Smolic and M. Smolic, "Giant Gravitons - with Strings Attached (II)," JHEP 0709 (2007) 049 [arXiv:hep-th/0701067]; D. Bekker, R. de Mello Koch and M. Stephanou, "Giant Gravitons - with Strings Attached (III)," JHEP 0802 (2008) 029 [arXiv:0710.5372 [hep-th]].

[12] D. Berenstein, D. H. Correa and S. E. Vazquez, "A study of open strings ending on giant gravitons, spin chains and integrability," JHEP 0609 (2006) 065 [arXiv:hep-th/0604123].

[13] H. Lin, O. Lunin and J. M. Maldacena, "Bubbling AdS space and 1/2 BPS geometries," JHEP 0410 (2004) 025 [arXiv:hep-th/0409174].

[14] Y. Kimura and S. Ramgoolam, "Branes, Anti-Branes and Brauer Algebras in GaugeGravity duality," JHEP 0711 (2007) 078 [arXiv:0709.2158 [hep-th]].

[15] T. W. Brown, P. J. Heslop and S. Ramgoolam, "Diagonal multi-matrix correlators and BPS operators in N=4 SYM," [arXiv:0711.0176 [hep-th]].

[16] R. Bhattacharyya, S. Collins and R. d. M. Koch, "Exact Multi-Matrix Correlators," JHEP 0803 (2008) 044 [arXiv:0801.2061 [hep-th]]; R. Bhattacharyya, R. d. M. Koch and M. Stephanou, "Exact Multi-Restricted Schur Polynomial Correlators," [arXiv:0805.3025 [hep-th]]. 
[17] T. Halverson, "Characters of the centralizer algebras of mixed tensor representations of $G L(r, \mathbb{C})$ and the quantum group $U_{q}(G L(r, \mathbb{C})$, " Pacific Journal of Mathematics, Vol. 174, No. $2,1996$.

[18] R. Goodman and N. Wallach, "Representations and Invariants of classical groups," CUP 1998.

[19] R. C. King, "Young tableaux, Schur functions and SU(2) plethysms," 1985 J. Phys. A: Math. Gen. 18 2429-2440.

[20] B. Feng, A. Hanany and Y. H. He, "Counting gauge invariants: The plethystic program," JHEP 0703 (2007) 090 [arXiv:hep-th/0701063].

[21] S. Dutta and R. Gopakumar, "Free Fermions and Thermal AdS/CFT," JHEP 0803 (2008) 011 [arXiv:0711.0133 [hep-th]].

[22] S. R. Das, A. Jevicki and S. D. Mathur, "Vibration modes of giant gravitons," Phys. Rev. D 63 (2001) 024013 [arXiv:hep-th/0009019].

[23] P. H. Ginsparg, "Applied Conformal Field Theory," [arXiv:hep-th/9108028].

[24] J. Polchinski, "String theory. Vol. 1: An introduction to the bosonic string," CUP (1998).

[25] T. Brown, R. de Mello Koch, S. Ramgoolam and N. Toumbas, "Correlators, probabilities and topologies in N = 4 SYM," JHEP 0703 (2007) 072 [arXiv:hep-th/0611290].

[26] N. Beisert, "The complete one-loop dilatation operator of $\mathrm{N}=4$ super Yang-Mills theory," Nucl. Phys. B 676 (2004) 3 [arXiv:hep-th/0307015].

[27] N. Beisert, M. Bianchi, J. F. Morales and H. Samtleben, "Higher spin symmetry and N = 4 SYM," JHEP 0407 (2004) 058 [arXiv:hep-th/0405057].

[28] S. E. Konstein, M. A. Vasiliev and V. N. Zaikin, "Conformal higher spin currents in any dimension and AdS/CFT correspondence," JHEP 0012 (2000) 018 [arXiv:hepth/0010239].

[29] W. Fulton and J. Harris, "Representation Theory: A First Course," Springer, 1991.

[30] M. Bianchi and V. Didenko, "Massive' higher spin multiplets and holography," [arXiv:hep-th/0502220].

[31] Koike and Terada, "Young Diagrammatic methods for the representation theory of the classical groups of type $B_{n}, C_{n}, D_{n}$, " Jour. of Algebra. 107, 466.

[32] C. Grood, "Brauer algebras and Centralizer algebras for $S O(2 n, \mathbb{C})$," Journal of Algebra 222, 678-707 (1999). 
[33] O. Aharony, J. Marsano, S. Minwalla, K. Papadodimas and M. Van Raamsdonk, "The Hagedorn / deconfinement phase transition in weakly coupled large N gauge theories," Adv. Theor. Math. Phys. 8 (2004) 603 [arXiv:hep-th/0310285].

[34] J. Kinney, J. M. Maldacena, S. Minwalla and S. Raju, "An index for 4 dimensional super conformal theories," Commun. Math. Phys. 275 (2007) 209 [arXiv:hep-th/0510251].

[35] F. A. Dolan, "Counting BPS operators in N=4 SYM," Nucl. Phys. B 790 (2008) 432 [arXiv:0704.1038 [hep-th]].

[36] V. Balasubramanian, M. x. Huang, T. S. Levi and A. Naqvi, "Open strings from N = 4 super Yang-Mills," JHEP 0208 (2002) 037 [arXiv:hep-th/0204196].

[37] B. . J. Stefanski and A. A. Tseytlin, "Large spin limits of AdS/CFT and generalized Landau-Lifshitz equations," JHEP 0405 (2004) 042 [arXiv:hep-th/0404133].

[38] S. S. Gubser, I. R. Klebanov and A. M. Polyakov, "A semi-classical limit of the gauge/string correspondence," Nucl. Phys. B 636 (2002) 99 [arXiv:hep-th/0204051].

[39] I. Biswas, D. Gaiotto, S. Lahiri and S. Minwalla, "Supersymmetric states of N = 4 Yang-Mills from giant gravitons," JHEP 0712 (2007) 006 [arXiv:hep-th/0606087].

[40] G. Mandal and N. V. Suryanarayana, "Counting 1/8-BPS dual-giants," JHEP 0703 (2007) 031 [arXiv:hep-th/0606088].

[41] M. M. Caldarelli and P. J. Silva, "Multi giant graviton systems, SUSY breaking and CFT," JHEP 0402 (2004) 052 [arXiv:hep-th/0401213].

[42] L. Grant, P. A. Grassi, S. Kim and S. Minwalla, "Comments on 1/16 BPS Quantum States and Classical Configurations," JHEP 0805 (2008) 049 [arXiv:0803.4183 [hep-th]].

[43] T. W. Brown, "Permutations and the Loop," JHEP 0806 (2008) 008 [arXiv:0801.2094 [hep-th]].

[44] J. A. Minahan and K. Zarembo, "The Bethe-ansatz for N = 4 super Yang-Mills," JHEP 0303 (2003) 013 [arXiv:hep-th/0212208].

[45] N. Beisert and M. Staudacher, "The N = 4 SYM integrable super spin chain," Nucl. Phys. B 670 (2003) 439 [arXiv:hep-th/0307042].

[46] S. Bellucci, P. Y. Casteill, J. F. Morales and C. Sochichiu, "Spin bit models from nonplanar N = 4 SYM," Nucl. Phys. B 699 (2004) 151 [arXiv:hep-th/0404066].

[47] R. A. Janik and M. Trzetrzelewski, "Supergravitons from one loop perturbative N=4 SYM," [arXiv:0712.2714 [hep-th]].

[48] M. Hamermesh, "Group Theory and its Applications to Physical Problems," AddisonWesley Publishing Company, 1962. 